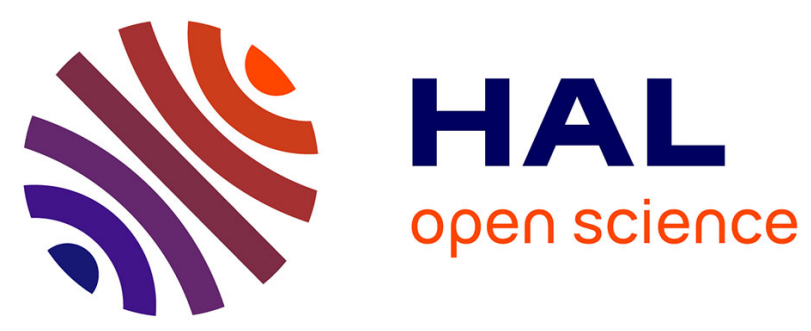

\title{
Competition between collective and noncollective excitation modes at high spin in $124 \mathrm{Ba}$
}

A. Al-Khatib, A.K. Singh, H. Hubel, P. Bringel, A. Burger, J. Domscheit, A. Neusser-Neffgen, G. Schoenwasser, G.B. Hagemann, C.R. Hansen, et al.

\section{- To cite this version:}

A. Al-Khatib, A.K. Singh, H. Hubel, P. Bringel, A. Burger, et al.. Competition between collective and noncollective excitation modes at high spin in 124Ba. Physical Review C, 2006, 74, pp.014305. 10.1103/PhysRevC.74.014305 . in2p3-00090258

\section{HAL Id: in2p3-00090258 https://hal.in2p3.fr/in2p3-00090258}

Submitted on 4 Jan 2007

HAL is a multi-disciplinary open access archive for the deposit and dissemination of scientific research documents, whether they are published or not. The documents may come from teaching and research institutions in France or abroad, or from public or private research centers.
L'archive ouverte pluridisciplinaire HAL, est destinée au dépôt et à la diffusion de documents scientifiques de niveau recherche, publiés ou non, émanant des établissements d'enseignement et de recherche français ou étrangers, des laboratoires publics ou privés. 


\title{
Competition between collective and noncollective excitation modes at high spin in ${ }^{124} \mathrm{Ba}$
}

\author{
A. Al-Khatib, A.K. Singh*, H. Hübel, P. Bringel, A. Bürger, \\ J. Domscheit, A. Neußer-Neffgen, and G. Schönwaßer \\ Helmholtz-Institut für Strahlen- und Kernphysik, \\ Universität Bonn, Nussallee 14-16, D-53115 Bonn, Germany
}

G.B. Hagemann, C. Ronn Hansen, B. Herskind, G. Sletten, and J.N. Wilson ${ }^{\dagger}$

Niels Bohr Institute, Blegdamsvej 17, DK-2100 Copenhagen Ø, Denmark

J. Timár, A. Algora, Zs. Dombrádi, J. Gál, G. Kalinka,

J. Molnár, B.M. Nyakó, D. Sohler, and L. Zolnai

Institute of Nuclear Research of the Hungarian

Academy of Sciences, H-4001 Debrecen, Hungary

R. Clark, M. Cromaz, P. Fallon, I.Y. Lee, A.O. Macchiavelli, and D. Ward Nuclear Science Division, Lawrence Berkeley National Laboratory, Berkeley CA 94720, USA

H. Amro $\ddagger$ and W.C. Ma

Department of Physics, Mississipi State University, Mississipi State, MS 39762, USA

M. Kmiecik, A. Maj, J. Styczen, and K. Zuber

Niewodniczanski Institute of Nuclear Physics, Polish Academy of Sciences, PL-31342 Krakow, Poland

K. Hauschild, A. Korichi, A. Lopez-Martens, J. Roccaz, and S. Siem ${ }^{\S}$ CSNSM Orsay, IN2P3/CNRS, F-91405 Orsay, France

F. Hannachi and J.N. Scheurer

Centre d'Etudes Nucléaires de Bordeaux-Gradignan, F-33175 Gradignan, France

P. Bednarczyk ${ }^{\Uparrow}$, Th. Byrski, D. Curien, O. Dorvaux, G.

\footnotetext{
* Present address: Department of Physics, IIT Kharagpur, Kharagpur - 721302, India

$\dagger$ Present address: LPSC/IN2P3, F-38026 Grenoble Cedex, France

$\ddagger$ Present address: Department of Physics, Univ. of Notre Dame, Notre Dame, IN 46556-5670, USA

$\S$ Permanent address: Department of Physics, University of Oslo, N-0316 Oslo, Norway

ฯ Permanent address: Niewodniczanski Institute of Nuclear Physics, Polish Academy of Sciences, PL-31342 Krakow, Poland. Present address: GSI, D-64291 Darmstadt, Germany
} 
Duchêne, B. Gall, F. Khalfallah, I. Piqueras, and J. Robin Institut de Recherches Subatomiques, CNRS-IN2P3, F-67037 Strasbourg, France

A. Görgen

DAPNIA/SPhN, CEA-Saclay, F-91191 Gif-sur-Yvette, France

K. Juhász

Department of Information Technology, Faculty of Informatics, University of Debrecen, H-4032 Debrecen, Hungary

S.B. Patel

Department of Physics, University of Mumbai, Mumbai, India

A.O. Evans and G. Rainovski

Oliver Lodge Laboratory, University of Liverpool, Liverpool L69 7ZE, UK

G. Benzoni, A. Bracco, F. Camera, S. Leoni, P. Mason, B. Million, A. Paleni, R. Sacchi, and O. Wieland Dipartimento di Fisica, Università degli Studi di Milano and INFN, Sezione di Milano, via Celoria 16, 20133 Milano, Italy

C.M. Petrache and D. Petrache

Dipartimento di Fisica, Università di Camerino and INFN,

Sezione di Perugia, I-62032 Camerino, Italy

G. La Rana and R. Moro

Physical Science Department and INFN,

Complesso Universitario di Monte S. Angelo, via Cinthia, I-80126 Napoli, Italy

G. De Angelis

Istituto Nazionale di Fisica Nucleare, Laboratori Nazionali de Legnaro, via Romea 4, I-35020 Legnaro, Italy

J.C. Lisle

Schuster Laboratory, University of Manchester, Brunswick Street, Manchester M13 9PL, UK

B. Cederwall and K. Lagergen 
Department of Physics, Royal Institute of Technology, SE-10691 Stockholm, Sweden

R.M. Lieder, E. Podsvirova, W. Gast, and H. Jäger

Institut für Kernphysik, Forschungszentrum Jülich, D-52425 Jülich, Germany

N. Redon

IPN Lyon, IN2P3/CNRS, Universitè de Lyon-1, F-69622 Villeurbanne, France

(Dated: February 9, 2006)

\begin{abstract}
High-spin states in ${ }^{124} \mathrm{Ba}$ were investigated in two experiments using the ${ }^{64} \mathrm{Ni}\left({ }^{64} \mathrm{Ni}, 4 \mathrm{n}\right){ }^{124} \mathrm{Ba}$ reaction at three different beam energies. In-beam $\gamma$-ray coincidences were measured with the Euroball and Gammasphere detector arrays, respectively. In the experiment with Euroball, the CsI detector array Diamant was employed to discriminate against charged-particle channels. Six new rotational bands were observed in ${ }^{124} \mathrm{Ba}$ and previously known bands were extended to higher spins and in the low-spin region. One of the bands shows a transition from collective to non-collective behavior at high spins. Configuration assignments are suggested on the basis of comparison with cranked shell model and cranked Nilsson-Strutinsky calculations.
\end{abstract}

PACS numbers: 23.20.Lv, 23.20.En, 27.60.+j, 21.60.Ev 


\section{INTRODUCTION}

Transitional nuclei between spherical and strongly deformed regions of the nuclear chart are usually soft with respect to deformation changes. Shape changes, including triaxiality, can be induced by the excitation of nucleons into specific deformation-driving orbitals. In the mass region around $A=125$, both protons and neutrons can occupy the unique-parity $h_{11 / 2}$ intruder orbitals which play an important role in driving the nuclear shape. The proton Fermi surface lies in the lower part of the $h_{11 / 2}$ subshell, which favors prolate shape, whereas the neutron Fermi surface lies in the middle or upper part of the $h_{11 / 2}$ subshell, which favors oblate shape. Thus, a coexistence of different shapes is expected in these nuclei due to opposite shape-driving forces of protons and neutrons in $h_{11 / 2}$ orbitals.

Several studies of the development of the shape and collectivity as a function of neutron and proton number have been made in this mass region, e.g. for Xe, Ba and Ce nuclei [1-18]. It was found that the deformation increases with increasing proton number towards $Z=66$ whereas it decreases with increasing neutron number towards the $N=82$ shell closure. Total routhian surface (TRS) calculations show that after the proton $h_{11 / 2}$ alignment the nuclei remain prolate $\left(\gamma \simeq 0^{\circ}\right)$ whereas the alignment of $h_{11 / 2}$ neutrons favors a triaxial shape with $\gamma \simeq-30^{\circ}[11,14]$.

For nuclei with a small number of particles outside the semi-closed core with $Z=50$ and $N=64$, the alignment of these particles along the rotation axis at high spin can polarize the nuclei towards oblate shape. In this spin range the rotational bands loose collectivity and single-particle alignments are favored as an efficient way to generate angular momentum. The collective bands terminate when all the single-particle angular momenta involved are aligned along the rotation axis. Higher-spin states can then be generated only by breaking the core.

Transitions from prolate to oblate shape have been observed to take place in two different ways, as smooth band termination, e.g. in the $A \simeq 110$ region [19], and as sudden band termination, e.g. in the $A \simeq 160$ region [20]. In the $A=125$ region the low-energy level structure is dominated by collective rotational bands with a moderate prolate deformation $\left(\varepsilon_{2} \simeq 0.25\right.$ and $\left.\gamma \simeq 0^{\circ}\right)$. However, non-collective excitations have been found to compete with collective rotation at high spin, e.g. in Xe [5] and Cs [21] isotopes, and may become yrast when the proton number increases. One of the aims of the present work was to explore such features in ${ }^{124} \mathrm{Ba}$. Eight rotational bands were previously known in this nucleus [12]. In this work, they were extended to much higher spins and partly also in the low-spin region. In addition, six new bands were discovered and a transition to non-collective excitations was observed in the yrast band. A preliminary report on this work has been given at a recent 
conference [22].

In the following section the experimental details and the data analysis are described, followed by a presentation of the experimental results and the level scheme in section III. In section IV the configurations of the observed structures are discussed and a brief summary is geven in the last section.

\section{EXPERIMENTAL DETAILS AND DATA ANALYSIS}

Two experiments were performed in which high-spin states in ${ }^{124} \mathrm{Ba}$ were populated using the ${ }^{64} \mathrm{Ni}\left({ }^{64} \mathrm{Ni}, 4 \mathrm{n}\right)$ reaction at beam energies of 255,261 and $265 \mathrm{MeV}$, respectively. The higher beam energies were chosen to enhance the population of very high-spin structures since the primary goal of both experiments was to search for hyperdeformation. The search for hyperdeformation is still in progress [23]. A number of different reaction channels are open at these beam energies, among them the $4 \mathrm{n}$ channel leading to ${ }^{124} \mathrm{Ba}$ which is the strongest of the neutron-evaporation channels. In this work, we focus on the normal-deformed states in this nucleus.

The first experiment was carried out at Lawrence Berkeley National Laboratory. The ${ }^{64} \mathrm{Ni}$ beam of $265 \mathrm{MeV}$ was provided by the 88-Inch cyclotron. Gamma-ray coincidences were measured using the Gammasphere spectrometer with 100 Compton-suppressed Ge detectors [24]. The target consisted of a ${ }^{64} \mathrm{Ni}$ foil of $476 \mu \mathrm{g} / \mathrm{cm}^{2}$ thickness, enriched to $96.5 \%$. Data were recorded with a trigger condition of six or more Ge detectors after Compton suppression and 15 or more 'modules' showing a signal. A module is defined as a Ge detector and the BGO scintillator of the suppression shields. After presorting and setting a prompt time window, a total of $1.2 \cdot 10^{9}$ events with Ge fold $\geqslant 4$ were obtained.

The second experiment was performed at the Institut de Recherches Subatomiques at Strasbourg. The ${ }^{64} \mathrm{Ni}$ beams with energies of 255 and $261 \mathrm{MeV}$ were provided by the Vivitron Tandem accelerator. A self supporting foil of $\simeq 500 \mu \mathrm{g} / \mathrm{cm}^{2}$ thickness was used as a target. Gamma-ray coincidences were measured using the Euroball spectrometer [25, 26]. It consisted of 30 Compton-suppressed conventional (tapered) Ge detectors and 41 composite Ge detectors. Of the latter, the 26 Clover detectors are composed of four Ge crystals each and the 15 Cluster detectors consist of seven Ge crystals each. At the time of the experiment, 230 individual Ge crystals out of the total of 239 of the full array could be used. An 'inner ball' of 210 BGO scintillation detectors was used as a multiplicity filter to enhance the selection of high-spin events. In addition, the charged-particle array Diamant [27, 28] consisting of 84 CsI scintillation detectors covering a geometrical solid angle of $95 \%$ was placed inside 
the target chamber. Each crystal has a size of $4.5 \times 4.5 \mathrm{~mm}^{2}$ and a thickness of $3 \mathrm{~mm}$. The CsI detectors were wrapped in $1.5 \mu \mathrm{m}$ thick aluminized mylar foil for light reflection. In front of the forward detectors ( up to $67.5^{\circ}$ ), Ta foils with thicknesses between 5 and $15 \mu \mathrm{m}$ were mounted to reduce the background from scattered beam particles and delta electrons. Coincidence events were recorded with the requirement that at least three Ge crystals and eleven BGO detectors of the inner ball were in coincidence.

In the off-line analysis, a presorting of the data which includes Compton suppression, pile-up rejection, gain matching and calibration of the Ge detectors was made. For the investigation of ${ }^{124} \mathrm{Ba}$ the information from the Diamant detectors was used to reject events in which charged particles were detected. After setting a wide gate on the prompt time peaks, $\sim 3$. $10^{9}$ events of three- and higher-fold Ge-detector concidences remained. The coincidence events from both experiments were unpacked and sorted off-line into two-, three- and fourdimensional arrays (matrices, cubes and hypercubes, respectively) using the RADWARE program package [29].

To obtain information on the multipole order of $\gamma$-ray transitions, an asymmetric matrix was sorted with events registered in the detectors at forward (average angle $35^{\circ}$ ) and backward (average angle $156^{\circ}$ ) angles on one axis and those detected near- $90^{\circ}$ on the other axis. Directional correlation (DCO) ratios, defined as $R_{D C O}=I\left(\gamma_{2}^{f b}, \gamma_{1}^{90^{\circ}}\right) / I\left(\gamma_{2}{ }^{90^{\circ}}, \gamma_{1}^{f b}\right)$, where $I\left(\gamma_{2}, \gamma_{1}\right)$ denotes the intensity of $\gamma_{2}$ in the spectrum gated on $\gamma_{1}$, were determined by setting gates on stretched E2 transitions. For a multipolarity determination of low-intensity transitions two angular correlation matrices were sorted. The first one contained events detected at forward and backward angles on one axis and those registered in all detectors on the other axis. Similarly, the second matrix contained events detected around $90^{\circ}$ on one axis and those of all detectors on the other axis. Gates were set in these matrices on the axis with events detected in all detectors. The intensity ratio $R_{A}=I\left(\gamma_{2}{ }^{f b}, \gamma_{1}^{\text {all }}\right) / I\left(\gamma_{2}{ }^{90^{\circ}}, \gamma_{1}^{\text {all }}\right)$ was used to distinguish between stretched dipole and stretched quadrupole transitions. Values of the DCO and the angular correlation ratios were around 0.6 and 1.0 for stretched dipole and stretched quadrupole transitions, respectively.

Linear-polarization measurements of $\gamma$ rays linking the various bands to levels of known spin and parity were made to determine the parity of the bands. The Clover detectors of the Euroball spectrometer were used for this purpose. The reference plane of each $\gamma$ ray is spanned by the beam axis and the emission direction of the $\gamma$ ray. Compton-scattered events in neighboring pairs of Ge crystals of the Clover detectors, scattered horizontally $(\mathrm{h})$ and vertically (v) to the reference plane, were analyzed separately. Two pairs of $E_{\gamma}-E_{\gamma}$ matrices were created with either horizontally or vertically scattered $\gamma$ rays incremented on one axis and $\gamma$ rays from all the detectors on the other axis. Such pairs of matrices were sorted with 
gates on uncontaminated $\gamma$-ray transitions from the bands. The linear-polarization asymmetry ratio, $A\left(E_{\gamma}\right)=\left[I_{v}\left(E_{\gamma}\right)-I_{h}\left(E_{\gamma}\right)\right] /\left[I_{v}\left(E_{\gamma}\right)+I_{h}\left(E_{\gamma}\right)\right]$, was obtained by setting gates on $\gamma$-ray transitions in the two matrices. Here, $I_{v}\left(E_{\gamma}\right)$ and $I_{h}\left(E_{\gamma}\right)$ are the intensities of vertically and horizontally scattered $\gamma$ rays of energy $E_{\gamma}$, respectively. The linear polarization, $P$, was calculated from this asymmetry ratio by $P=A\left(E_{\gamma}\right) Q\left(E_{\gamma}\right)$, where $Q\left(E_{\gamma}\right)$ is the polarization sensitivity of the polarimeter. For point-like detectors this quantity can be calculated using the Klein-Nishina formula [30]. For finite crystal-size detectors it was obtained by introducing a scaling factor. The scaling factor has been measured for the Euroball array in several experiments. We have adopted the linear-polarization sensitivity obtained in ref. [31] for our analysis. The linear-polarization asymmetry for pure stretched magnetic transitions is negative, whereas for pure stretched electric transitions it is positive.

\section{RESULTS AND LEVEL SCHEME}

The energy-level scheme of ${ }^{124} \mathrm{Ba}$ is presented in Fig. 1. It is based on the results of the present work except for the lower-spin part which has been adopted from previous work $[12,18,32]$. Eight of the fourteen rotational bands were known previously. They were partly extended at low and high spins. The level scheme was constructed on the basis of coincidence relationships and relative $\gamma$-ray intensities. Spin assignments are based on DCO and angular correlation ratios. For transitions between low-spin states, no angular-correlation information could be obtained due to the loss of the spin alignment from the reaction. While the alignment stays approximately constant at high spins, it is reduced at low spins by the interaction between the nuclear moments and the fields produced by the atomic electrons. Transition energies and their relative intensities, DCO ratios, multipolarities and placements in the level scheme are listed in Table I. In several cases, in particular for Bands 12 and 13, it was not possible to obtain reliable intensities since connecting transitions to bands with well established intensities were too weak. However, in some of these cases DCO or angular correlation ratios could be determined, as they are derived from intensity ratios.

Band 1 was already established in previous work [12] up to the $I^{\pi}=34^{+}$state. Above this level, we observe several new transitions resulting in an irregular level pattern. Fig. 2 shows two $\gamma$-ray coincidence spectra which demonstrate the forking of Band 1 above the $1444.3 \mathrm{keV}$ transition and into the 1583.4 - $1610.9 \mathrm{keV}$ sequence. The DCO ratio of the $1444.3 \mathrm{keV}$ transition is compatible with a stretched quadrupole, presumably E2, transition. The multipolarity of the other new transitions could not be determined. Hence, the spin assignments to the high-spin levels are tentative and, therefore, given in parenthesis in Fig. 1. 


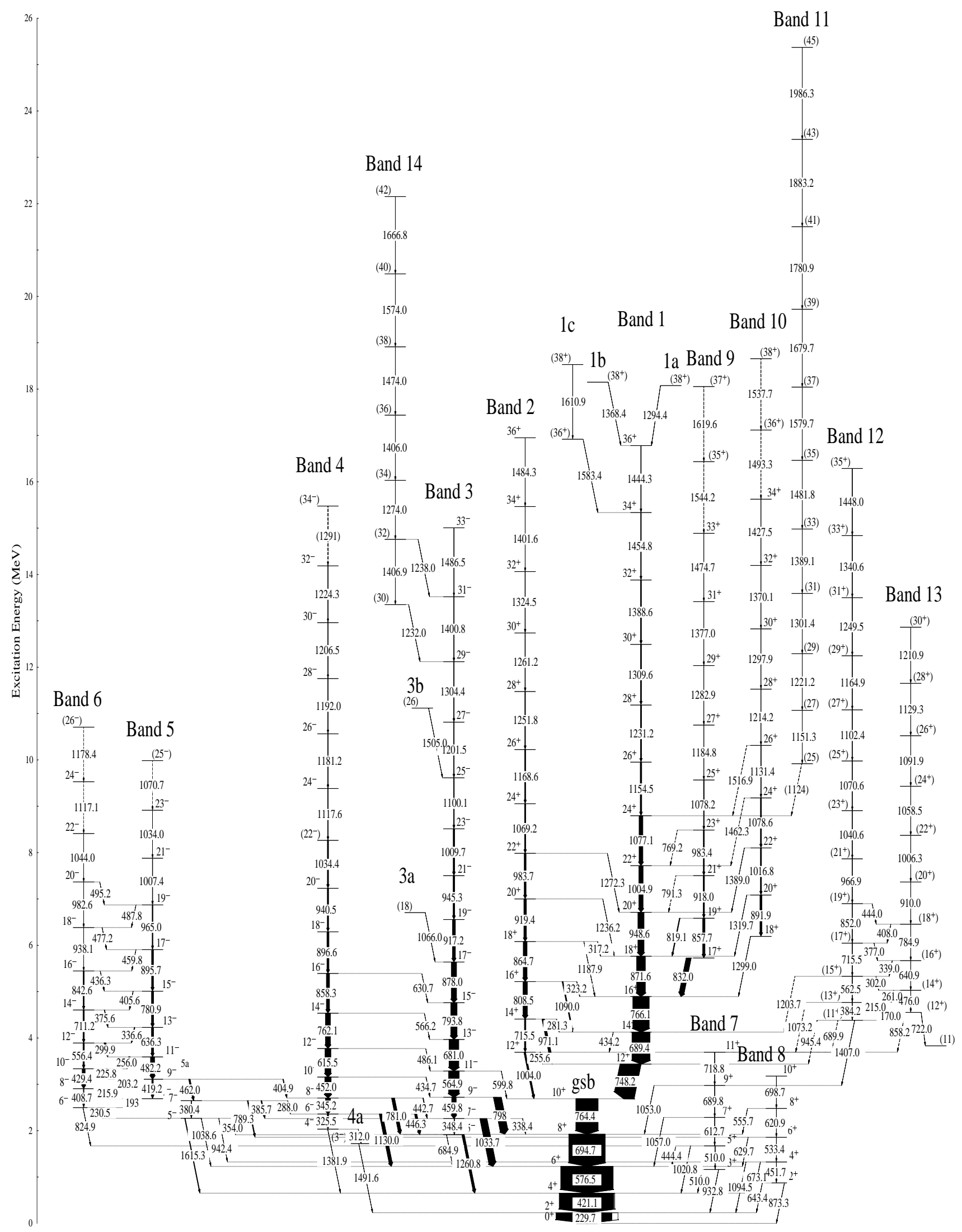

FIG. 1: Level scheme of ${ }^{124} \mathrm{Ba}$ based on present work and previous results [12]. Transition energies are given in $\mathrm{keV}$ and the widths of the arrows are proportional to relative $\gamma$-ray intensities. 
Band 2, which was known up to the $I^{\pi}=24^{+}$state [12], is extended by six transitions to higher spins. For the new transitions stretched quadrupole, probably E2, multipolarity was established up to $1261.2 \mathrm{keV}$. However, due to the regularity of the band, E2 multipolarity may be assumed also for the other high-spin transitions. Several new inter-band transitions connecting Band 2 with Band 1 have also been observed.

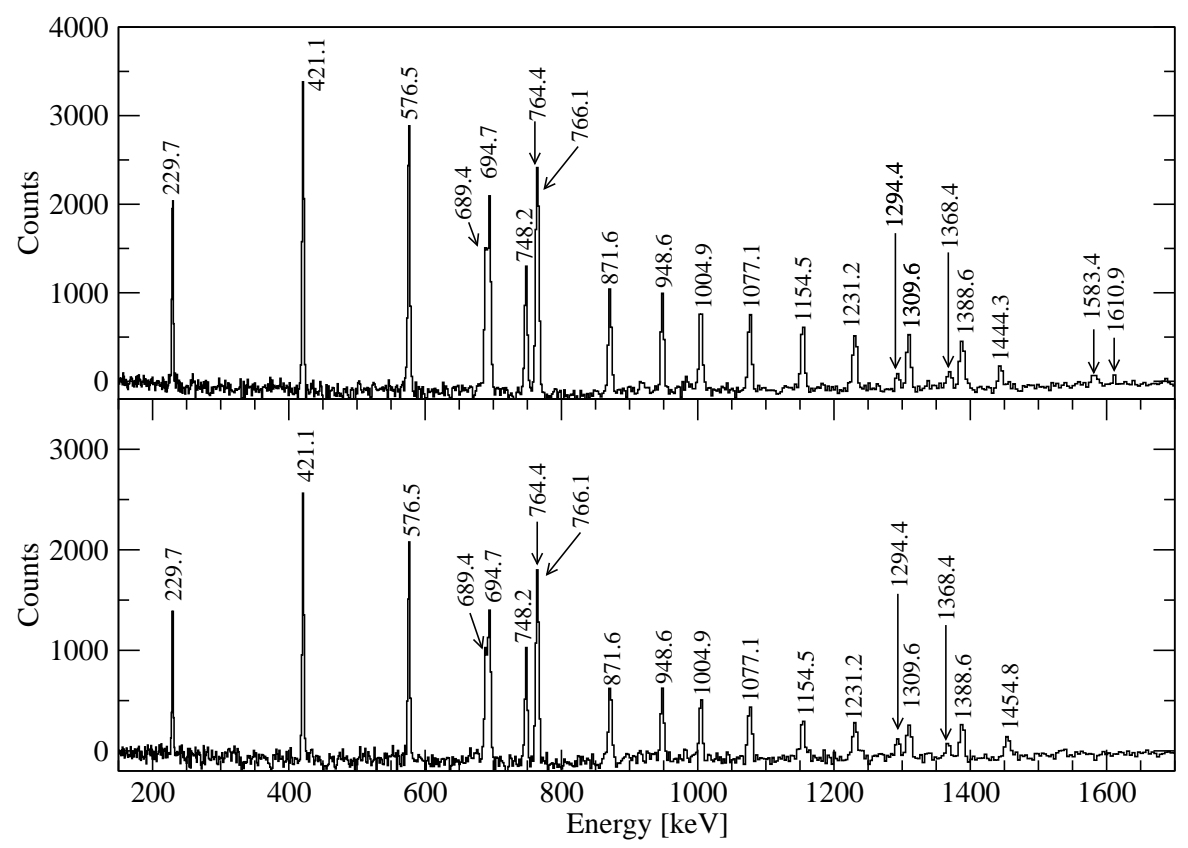

FIG. 2: Summed triple-gated $\gamma$-ray coincidence spectra of Band 1. The upper spectrum was produced by setting two gates on transitions (689.4 to $1444.3 \mathrm{keV}$ ) of Band 1 and one gate on the $1454.8 \mathrm{keV}$ transition. The lower spectrum was obtained by setting two gates on transitions (689.4 to $1454.8 \mathrm{keV}$ ) of Band 1 and one gate on the $1444.3 \mathrm{keV}$ transition. 
TABLE I: Energies, relative intensities, DCO ratios, multipolarity and spin assignments of $\gamma$-ray transitons of ${ }^{124} \mathrm{Ba}$

Excitation Energy $^{a}$ Intensity $^{b} R_{D C O}^{c}$ Band $_{i} \rightarrow$ Band $_{f}$ Assignment Multipolarity

$$
E_{i}(\mathrm{keV}) \quad E_{\gamma}(\mathrm{keV}) \quad I_{\gamma} \quad \text { ratio } \quad J_{i}^{\pi} \rightarrow J_{f}^{\pi}
$$

\begin{tabular}{ccccccc}
\hline \hline 230 & 229.7 & $536(31)$ & - & $g s b \rightarrow g s b$ & $2^{+} \rightarrow 0^{+}$ & E2 \\
652 & 421.1 & $524(28)$ & - & $g s b \rightarrow g s b$ & $4^{+} \rightarrow 2^{+}$ & E2 \\
873 & 643.4 & - & - & $8 \rightarrow g s b$ & $2^{+} \rightarrow 2^{+}$ & M1 \\
873 & 873.3 & - & - & $8 \rightarrow g s b$ & $2^{+} \rightarrow 0^{+}$ & E2 \\
1162 & 510.0 & - & - & $7 \rightarrow g s b$ & $3^{+} \rightarrow 4^{+}$ & M1 \\
1162 & 932.8 & - & - & $7 \rightarrow g s b$ & $3^{+} \rightarrow 2^{+}$ & M1 \\
1228 & 576.5 & 500 & 0.90 & $g s b \rightarrow g s b$ & $6^{+} \rightarrow 4^{+}$ & E2 \\
1325 & 451.7 & - & - & $8 \rightarrow 8$ & $4^{+} \rightarrow 2^{+}$ & E2 \\
1325 & 673.1 & - & - & $8 \rightarrow g s b$ & $4^{+} \rightarrow 4^{+}$ & M1 \\
1325 & 1094.5 & - & & $8 \rightarrow g s b$ & $4^{+} \rightarrow 2^{+}$ & E2 \\
1672 & 444.4 & - & - & $7 \rightarrow g s b$ & $5^{+} \rightarrow 6^{+}$ & M1 \\
1672 & 510.0 & - & - & $7 \rightarrow 7$ & $5^{+} \rightarrow 3^{+}$ & E2 \\
1672 & 1020.8 & - & - & $7 \rightarrow g s b$ & $5^{+} \rightarrow 4^{+}$ & M1 \\
1722 & 1491.6 & - & - & $4 a \rightarrow g s b$ & $\left(3^{-}\right) \rightarrow 2^{+}$ & E1 \\
1858 & 533.4 & - & - & $8 \rightarrow 8$ & $6^{+} \rightarrow 4^{+}$ & E2 \\
1858 & 629.7 & - & - & $8 \rightarrow g s b$ & $6^{+} \rightarrow 6^{+}$ & M1 \\
1913 & 684.9 & - & - & $3 \rightarrow g s b$ & $5^{-} \rightarrow 6^{+}$ & E1 \\
1913 & 1260.8 & $23(5)$ & 0.74 & $3 \rightarrow g s b$ & $5^{-} \rightarrow 4^{+}$ & E1 \\
1923 & 694.7 & $348(22)$ & 1.00 & $g s b \rightarrow g s b$ & $8^{+} \rightarrow 6^{+}$ & E2 \\
2034 & 312.0 & - & 0.45 & $4 \rightarrow 4 a$ & $4^{-} \rightarrow\left(3^{-}\right)$ & M1 \\
2034 & 1381.9 & - & 1.11 & $4 \rightarrow g s b$ & $4^{-} \rightarrow 4^{+}$ & E1 \\
2262 & 338.4 & $2(1)$ & - & $3 \rightarrow g s b$ & $7^{-} \rightarrow 8^{+}$ & E1 \\
2262 & 348.4 & $6(1)$ & - & $3 \rightarrow 3$ & $7^{-} \rightarrow 5^{-}$ & E2 \\
\hline
\end{tabular}


TABLE I: (continued)

Excitation Energy ${ }^{a}$ Intensity $^{b} R_{D C O}^{c}$ Band $_{i} \rightarrow$ Band $_{f}$ Assignment Multipolarity $E_{i}(\mathrm{keV}) \quad E_{\gamma}(\mathrm{keV}) \quad I_{\gamma} \quad$ ratio $\quad J_{i}^{\pi} \rightarrow J_{f}^{\pi}$

\begin{tabular}{ccccccc}
\hline \hline 2262 & 1033.7 & $67(16)$ & 0.66 & $3 \rightarrow g s b$ & $7^{-} \rightarrow 6^{+}$ & E1 \\
2267 & 354.0 & - & - & $5 a \rightarrow 3$ & $5^{-} \rightarrow 5^{-}$ & M1 \\
2267 & 942.4 & - & - & $5 a \rightarrow 8$ & $5^{-} \rightarrow 4^{+}$ & E1 \\
2267 & 1038.6 & - & - & $5 a \rightarrow g s b$ & $5^{-} \rightarrow 6^{+}$ & E1 \\
2267 & 1615.3 & $6(2)$ & - & $5 a \rightarrow g s b$ & $5^{-} \rightarrow 4^{+}$ & E1 \\
2285 & 612.7 & - & - & $7 \rightarrow 7$ & $7^{+} \rightarrow 5^{+}$ & E2 \\
2285 & 1057.0 & - & - & $7 \rightarrow g s b$ & $7^{+} \rightarrow 6^{+}$ & M1 \\
2359 & 325.5 & $13(2)$ & 1.32 & $4 \rightarrow 4$ & $6^{-} \rightarrow 4^{-}$ & E2 \\
2359 & 446.3 & $22(4)$ & 0.35 & $4 \rightarrow 3$ & $6^{-} \rightarrow 5^{-}$ & M1 \\
2359 & 1130.0 & $31(7)$ & 0.99 & $4 \rightarrow g s b$ & $6^{-} \rightarrow 6^{+}$ & E1 \\
2479 & 555.7 & - & - & $8 \rightarrow g s b$ & $8^{+} \rightarrow 8^{+}$ & M1 \\
2479 & 620.9 & - & - & $8 \rightarrow 8$ & $8^{+} \rightarrow 6^{+}$ & E2 \\
2498 & 230.5 & $64(12)$ & 0.58 & $6 \rightarrow 5 a$ & $6^{-} \rightarrow 5^{-}$ & M1 \\
2498 & 824.9 & - & - & $6 \rightarrow 7$ & $6^{-} \rightarrow 5^{+}$ & E1 \\
2647 & 288.0 & $2(1)$ & - & $5 a \rightarrow 4$ & $7^{-} \rightarrow 6^{-}$ & M1 \\
2647 & 380.4 & $5(2)$ & 1.05 & $5 a \rightarrow 5 a$ & $7^{-} \rightarrow 5^{-}$ & E2 \\
2647 & 385.7 & $7(2)$ & 0.67 & $5 a \rightarrow 3$ & $7^{-} \rightarrow 7^{-}$ & M1 \\
2647 & 789.3 & $8(3)$ & - & $5 a \rightarrow 8$ & $7^{-} \rightarrow 6^{+}$ & E1 \\
2688 & 764.4 & $213(32)$ & 0.97 & $g s b \rightarrow g s b$ & $10^{+} \rightarrow 8^{+}$ & E2 \\
2691 & 193.0 & $39(14)$ & 0.41 & $5 \rightarrow 6$ & $7^{-} \rightarrow 6^{-}$ & M1 \\
2705 & 345.2 & $46(8)$ & 0.99 & $4 \rightarrow 4$ & $8^{-} \rightarrow 6^{-}$ & E2 \\
2705 & 442.7 & $6(3)$ & 0.48 & $4 \rightarrow 3$ & $8^{-} \rightarrow 7^{-}$ & M1 \\
2705 & 781.0 & $29(6)$ & 0.75 & $4 \rightarrow g s b$ & $8^{-} \rightarrow 8^{+}$ & E1 \\
\hline & 459.8 & $43(9)$ & 1.13 & $3 \rightarrow 3$ & $9^{-} \rightarrow 7^{-}$ & E2 \\
\hline
\end{tabular}


TABLE I: (continued)

Excitation Energy ${ }^{a}$ Intensity $^{b} R_{D C O}^{c}$ Band $_{i} \rightarrow$ Band $_{f}$ Assignment Multipolarity $E_{i}(\mathrm{keV}) \quad E_{\gamma}(\mathrm{keV}) \quad I_{\gamma} \quad$ ratio $\quad J_{i}^{\pi} \rightarrow J_{f}^{\pi}$

\begin{tabular}{|c|c|c|c|c|c|c|}
\hline 2722 & 798.0 & $75(8)$ & 0.50 & $3 \rightarrow g s b$ & $9^{-} \rightarrow 8^{+}$ & E1 \\
\hline 2906 & 215.9 & $23(11)$ & 0.35 & $6 \rightarrow 5$ & $8^{-} \rightarrow 7^{-}$ & M1 \\
\hline 2906 & 408.7 & $6(4)$ & - & $6 \rightarrow 6$ & $8^{-} \rightarrow 6^{-}$ & $\mathrm{E} 2$ \\
\hline 2975 & 689.8 & - & - & $7 \rightarrow 7$ & $9^{+} \rightarrow 7^{+}$ & $\mathrm{E} 2$ \\
\hline 2975 & 1053.0 & - & - & $7 \rightarrow g s b$ & $9^{+} \rightarrow 8^{+}$ & M1 \\
\hline 3110 & 203.2 & - & - & $5 \rightarrow 6$ & $9^{-} \rightarrow 8^{-}$ & M1 \\
\hline 3110 & 404.9 & $6(2)$ & 0.77 & $5 \rightarrow 4$ & $9^{-} \rightarrow 8^{-}$ & M1 \\
\hline 3110 & 419.2 & $15(3)$ & - & $5 \rightarrow 5$ & $9^{-} \rightarrow 7^{-}$ & E2 \\
\hline 3110 & 462.0 & $14(5)$ & 0.90 & $5 \rightarrow 5 a$ & $9^{-} \rightarrow 7^{-}$ & $\mathrm{E} 2$ \\
\hline 3157 & 434.7 & $3(1)$ & - & $4 \rightarrow 3$ & $10^{-} \rightarrow 9^{-}$ & M1 \\
\hline 3157 & 452.0 & $61(17)$ & 1.02 & $4 \rightarrow 4$ & $10^{-} \rightarrow 8^{-}$ & $\mathrm{E} 2$ \\
\hline 3178 & 698.7 & - & - & $8 \rightarrow 8$ & $10^{+} \rightarrow 8^{+}$ & E2 \\
\hline 3287 & 564.9 & $101(22)$ & 1.09 & $3 \rightarrow 3$ & $11^{-} \rightarrow 9^{-}$ & E2 \\
\hline 3287 & 599.8 & - & - & $3 \rightarrow g s b$ & $11^{-} \rightarrow 10^{+}$ & E1 \\
\hline 3335 & 225.8 & $13(3)$ & - & $6 \rightarrow 5$ & $10^{-} \rightarrow 9^{-}$ & M1 \\
\hline 3335 & 429.4 & $28(6)$ & 1.01 & $6 \rightarrow 6$ & $10^{-} \rightarrow 8^{-}$ & E2 \\
\hline 3437 & 748.3 & 196(13) & 0.97 & $1 \rightarrow g s b$ & $12^{+} \rightarrow 10^{+}$ & E2 \\
\hline 3591 & 256.0 & $13(5)$ & - & $5 \rightarrow 6$ & $11^{-} \rightarrow 10^{-}$ & M1 \\
\hline 3591 & 482.2 & $39(8)$ & 0.95 & $5 \rightarrow 5$ & $11^{-} \rightarrow 9^{-}$ & $\mathrm{E} 2$ \\
\hline 3692 & 255.6 & $6(3)$ & 0.74 & $2 \rightarrow 1$ & $12^{+} \rightarrow 12^{+}$ & M1 \\
\hline 3692 & 1004.0 & $16(5)$ & 1.17 & $2 \rightarrow g s b$ & $12^{+} \rightarrow 10^{+}$ & E2 \\
\hline 3694 & 718.8 & - & - & $7 \rightarrow 7$ & $11^{+} \rightarrow 9^{+}$ & E2 \\
\hline 3772 & 486.1 & - & - & $4 \rightarrow 3$ & $12^{-} \rightarrow 11^{-}$ & M1 \\
\hline 3772 & 615.5 & $56(16)$ & 1.03 & $4 \rightarrow 4$ & $12^{-} \rightarrow 10^{-}$ & E2 \\
\hline
\end{tabular}


TABLE I: (continued)

Excitation Energy $^{a}$ Intensity $^{b} R_{D C O}^{c}$ Band $_{i} \rightarrow$ Band $_{f}$ Assignment Multipolarity $E_{i}(\mathrm{keV}) \quad E_{\gamma}(\mathrm{keV}) \quad I_{\gamma} \quad$ ratio $\quad J_{i}^{\pi} \rightarrow J_{f}^{\pi}$

\begin{tabular}{|c|c|c|c|c|c|c|}
\hline 3891 & 299.9 & $16(4)$ & - & $6 \rightarrow 5$ & $12^{-} \rightarrow 11^{-}$ & M1 \\
\hline 3891 & 556.4 & $13(5)$ & 0.97 & $6 \rightarrow 6$ & $12^{-} \rightarrow 10^{-}$ & E2 \\
\hline 3968 & 681.0 & $94(25)$ & 1.15 & $3 \rightarrow 3$ & $13^{-} \rightarrow 11^{-}$ & E2 \\
\hline 4127 & 434.2 & - & - & $1 \rightarrow 2$ & $14^{+} \rightarrow 12^{+}$ & E2 \\
\hline 4127 & 689.4 & $173(13)$ & 1.03 & $1 \rightarrow 1$ & $14^{+} \rightarrow 12^{+}$ & E2 \\
\hline 4228 & 336.6 & $7(2)$ & - & $5 \rightarrow 6$ & $13^{-} \rightarrow 12^{-}$ & M1 \\
\hline 4228 & 636.3 & $20(4)$ & 1.15 & $5 \rightarrow 5$ & $13^{-} \rightarrow 11^{-}$ & E2 \\
\hline 4382 & 689.9 & - & - & $12 \rightarrow 2$ & $\left(11^{+}\right) \rightarrow 12^{+}$ & M1 \\
\hline 4382 & 945.4 & - & - & $12 \rightarrow 1$ & $\left(11^{+}\right) \rightarrow 12^{+}$ & M1 \\
\hline 4382 & 1407.0 & - & - & $12 \rightarrow 7$ & $\left(11^{+}\right) \rightarrow 9^{+}$ & E2 \\
\hline 4408 & 281.3 & $3(1)$ & 0.53 & $2 \rightarrow 1$ & $14^{+} \rightarrow 14^{+}$ & M1 \\
\hline 4408 & 715.5 & $14(4)$ & 1.22 & $2 \rightarrow 2$ & $14^{+} \rightarrow 12^{+}$ & E2 \\
\hline 4408 & 971.1 & $24(6)$ & 1.38 & $2 \rightarrow 1$ & $14^{+} \rightarrow 12^{+}$ & E2 \\
\hline 4534 & 566.2 & - & - & $4 \rightarrow 3$ & $14^{-} \rightarrow 13^{-}$ & M1 \\
\hline 4534 & 762.1 & $48(16)$ & 1.03 & $4 \rightarrow 4$ & $14^{-} \rightarrow 12^{-}$ & E2 \\
\hline 4552 & 170.0 & - & - & $13 \rightarrow 12$ & $\left(12^{+}\right) \rightarrow\left(11^{+}\right)$ & M1 \\
\hline 4552 & 722.0 & - & 0.74 & $13 \rightarrow 13 a$ & $\left(12^{+}\right) \rightarrow(11)$ & - \\
\hline 4552 & 858.2 & - & - & $13 \rightarrow 7$ & $\left(12^{+}\right) \rightarrow 11^{+}$ & M1 \\
\hline 4604 & 375.6 & $5(2)$ & - & $6 \rightarrow 5$ & $14^{-} \rightarrow 13^{-}$ & M1 \\
\hline 4604 & 711.2 & $13(6)$ & 1.00 & $6 \rightarrow 6$ & $14^{-} \rightarrow 12^{-}$ & E2 \\
\hline 4762 & 793.8 & $61(9)$ & 1.03 & $3 \rightarrow 3$ & $15^{-} \rightarrow 13^{-}$ & E2 \\
\hline 4767 & 215.0 & - & - & $12 \rightarrow 13$ & $\left(13^{+}\right) \rightarrow\left(12^{+}\right)$ & M1 \\
\hline 4767 & 384.2 & - & 1.14 & $12 \rightarrow 12$ & $\left(13^{+}\right) \rightarrow\left(11^{+}\right)$ & E2 \\
\hline 4767 & 1073.2 & - & - & $12 \rightarrow 7$ & $\left(13^{+}\right) \rightarrow 11^{+}$ & E2 \\
\hline
\end{tabular}


TABLE I: (continued)

Excitation Energy $^{a}$ Intensity $^{b} R_{D C O}^{c}$ Band $_{i} \rightarrow$ Band $_{f}$ Assignment Multipolarity $E_{i}(\mathrm{keV}) \quad E_{\gamma}(\mathrm{keV}) \quad I_{\gamma} \quad$ ratio $\quad J_{i}^{\pi} \rightarrow J_{f}^{\pi}$

\begin{tabular}{ccccccc}
\hline \hline 4893 & 766.1 & $156(13)$ & 1.05 & $1 \rightarrow 1$ & $16^{+} \rightarrow 14^{+}$ & E2 \\
5010 & 405.6 & $3(1)$ & - & $5 \rightarrow 6$ & $15^{-} \rightarrow 14^{-}$ & M1 \\
5010 & 780.9 & $20(3)$ & 0.92 & $5 \rightarrow 5$ & $15^{-} \rightarrow 13^{-}$ & E2 \\
5028 & 261.0 & - & - & $13 \rightarrow 12$ & $\left(14^{+}\right) \rightarrow\left(13^{+}\right)$ & M1 \\
5028 & 476.0 & - & 1.05 & $13 \rightarrow 13$ & $\left(14^{+}\right) \rightarrow\left(12^{+}\right)$ & E2 \\
5216 & 323.2 & $3(2)$ & 0.78 & $2 \rightarrow 1$ & $16^{+} \rightarrow 16^{+}$ & M1 \\
5216 & 808.5 & $42(8)$ & 1.35 & $2 \rightarrow 2$ & $16^{+} \rightarrow 14^{+}$ & E2 \\
5216 & 1090.0 & $11(5)$ & - & $2 \rightarrow 1$ & $16^{+} \rightarrow 14^{+}$ & E2 \\
5330 & 302.0 & - & - & $12 \rightarrow 13$ & $\left(15^{+}\right) \rightarrow\left(14^{+}\right)$ & M1 \\
5330 & 562.5 & - & 1.03 & $12 \rightarrow 12$ & $\left(15^{+}\right) \rightarrow\left(13^{+}\right)$ & E2 \\
5330 & 1203.7 & - & - & $12 \rightarrow 1$ & $\left(15^{+}\right) \rightarrow 14^{+}$ & M1 \\
5392 & 630.7 & - & - & $4 \rightarrow 3$ & $16^{-} \rightarrow 15^{-}$ & M1 \\
5392 & 858.3 & $31(11)$ & 0.94 & $4 \rightarrow 4$ & $16^{-} \rightarrow 14^{-}$ & E2 \\
5446 & 436.3 & $2(1)$ & - & $6 \rightarrow 5$ & $16^{-} \rightarrow 15^{-}$ & M1 \\
5446 & 842.6 & $9(2)$ & 0.85 & $6 \rightarrow 6$ & $16^{-} \rightarrow 14^{-}$ & E2 \\
5639 & 878.0 & $51(12)$ & 1.00 & $3 \rightarrow 3$ & $17^{-} \rightarrow 15^{-}$ & E2 \\
5669 & 339.0 & - & - & $13 \rightarrow 12$ & $\left(16^{+}\right) \rightarrow\left(15^{+}\right)$ & M1 \\
5669 & 640.9 & - & 1.00 & $13 \rightarrow 13$ & $\left(16^{+}\right) \rightarrow\left(14^{+}\right)$ & E2 \\
5726 & 832.0 & $48(9)$ & 0.27 & $9 \rightarrow 1$ & $17^{+} \rightarrow 16^{+}$ & M1 \\
5764 & 871.6 & $84(15)$ & 1.10 & $1 \rightarrow 1$ & $18^{+} \rightarrow 16^{+}$ & E2 \\
5906 & 459.8 & - & - & $5 \rightarrow 6$ & $17^{-} \rightarrow 16^{-}$ & M1 \\
5906 & 895.7 & $18(3)$ & 1.12 & $5 \rightarrow 5$ & $17^{-} \rightarrow 15^{-}$ & E2 \\
6046 & 377.0 & - & - & $12 \rightarrow 13$ & $\left(17^{+}\right) \rightarrow\left(16^{+}\right)$ & M1 \\
\hline & 715.5 & - & 1.01 & $12 \rightarrow 12$ & $\left(17^{+}\right) \rightarrow\left(15^{+}\right)$ & E2 \\
\hline
\end{tabular}


TABLE I: (continued)

Excitation Energy ${ }^{a}$ Intensity $^{b} R_{D C O}^{c}$ Band $_{i} \rightarrow$ Band $_{f}$ Assignment Multipolarity $E_{i}(\mathrm{keV}) \quad E_{\gamma}(\mathrm{keV}) \quad I_{\gamma} \quad$ ratio $\quad J_{i}^{\pi} \rightarrow J_{f}^{\pi}$

\begin{tabular}{|c|c|c|c|c|c|c|}
\hline 6081 & 317.2 & - & - & $2 \rightarrow 1$ & $18^{+} \rightarrow 18^{+}$ & M1 \\
\hline 6081 & 864.7 & $37(9)$ & 0.93 & $2 \rightarrow 2$ & $18^{+} \rightarrow 16^{+}$ & E2 \\
\hline 6081 & 1187.9 & - & - & $2 \rightarrow 1$ & $18^{+} \rightarrow 16^{+}$ & E2 \\
\hline 6192 & 1299.0 & - & & $10 \rightarrow 1$ & $18^{+} \rightarrow 16^{+}$ & E2 \\
\hline 6290 & 896.6 & $20(8)$ & 1.21 & $4 \rightarrow 4$ & $18^{-} \rightarrow 16^{-}$ & E2 \\
\hline 6383 & 477.2 & - & - & $6 \rightarrow 5$ & $18^{-} \rightarrow 17^{-}$ & M1 \\
\hline 6383 & 938.1 & $9(2)$ & - & $6 \rightarrow 6$ & $18^{-} \rightarrow 16^{-}$ & E2 \\
\hline 6454 & 408.0 & - & - & $13 \rightarrow 12$ & $\left(18^{+}\right) \rightarrow\left(17^{+}\right)$ & M1 \\
\hline 6454 & 784.9 & - & 1.04 & $13 \rightarrow 13$ & $\left(18^{+}\right) \rightarrow\left(16^{+}\right)$ & E2 \\
\hline 6556 & 917.3 & $29(6)$ & 1.15 & $3 \rightarrow 3$ & $19^{-} \rightarrow 17^{-}$ & E2 \\
\hline 6583 & 819.1 & $7(2)$ & - & $9 \rightarrow 1$ & $19^{+} \rightarrow 18^{+}$ & M1 \\
\hline 6583 & 857.7 & $22(9)$ & 0.95 & $9 \rightarrow 9$ & $19^{+} \rightarrow 17^{+}$ & E2 \\
\hline 6705 & 1066.0 & - & - & $3 a \rightarrow 3$ & $(18) \rightarrow 17^{-}$ & - \\
\hline 6712 & 948.6 & $59(13)$ & 1.06 & $1 \rightarrow 1$ & $20^{+} \rightarrow 18^{+}$ & E2 \\
\hline 6871 & 487.8 & - & - & $5 \rightarrow 6$ & $19^{-} \rightarrow 18^{-}$ & M1 \\
\hline 6871 & 965.0 & $7(2)$ & 0.96 & $5 \rightarrow 5$ & $19^{-} \rightarrow 17^{-}$ & $\mathrm{E} 2$ \\
\hline 6898 & 444.0 & - & - & $12 \rightarrow 13$ & $\left(19^{+}\right) \rightarrow\left(18^{+}\right)$ & M1 \\
\hline 6898 & 852.0 & - & 1.06 & $12 \rightarrow 12$ & $\left(19^{+}\right) \rightarrow\left(17^{+}\right)$ & E2 \\
\hline 7000 & 919.4 & $25(6)$ & 1.08 & $2 \rightarrow 2$ & $20^{+} \rightarrow 18^{+}$ & E2 \\
\hline 7000 & 1236.2 & - & - & $2 \rightarrow 1$ & $20^{+} \rightarrow 18^{+}$ & E2 \\
\hline 7084 & 891.9 & $20(10)$ & 0.91 & $10 \rightarrow 10$ & $20^{+} \rightarrow 18^{+}$ & E2 \\
\hline 7084 & 1319.7 & - & 0.92 & $10 \rightarrow 1$ & $20^{+} \rightarrow 18^{+}$ & E2 \\
\hline 7230 & 940.5 & $13(6)$ & 0.90 & $4 \rightarrow 4$ & $20^{-} \rightarrow 18^{-}$ & E2 \\
\hline 7365 & 910.0 & - & 1.08 & $13 \rightarrow 13$ & $\left(20^{+}\right) \rightarrow\left(18^{+}\right)$ & $\mathrm{E} 2$ \\
\hline
\end{tabular}


TABLE I: (continued)

Excitation Energy $^{a}$ Intensity $^{b} R_{D C O}^{c}$ Band $_{i} \rightarrow$ Band $_{f}$ Assignment Multipolarity $E_{i}(\mathrm{keV}) \quad E_{\gamma}(\mathrm{keV}) \quad I_{\gamma} \quad$ ratio $\quad J_{i}^{\pi} \rightarrow J_{f}^{\pi}$

\begin{tabular}{|c|c|c|c|c|c|c|}
\hline 7366 & 495.2 & - & - & $6 \rightarrow 5$ & $20^{-} \rightarrow 19^{-}$ & M1 \\
\hline 7366 & 982.6 & $4(2)$ & - & $6 \rightarrow 6$ & $20^{-} \rightarrow 18^{-}$ & E2 \\
\hline 7502 & 945.3 & $16(4)$ & 1.26 & $3 \rightarrow 3$ & $21^{-} \rightarrow 19^{-}$ & E2 \\
\hline 7503 & 791.3 & - & - & $9 \rightarrow 1$ & $21^{+} \rightarrow 20^{+}$ & M1 \\
\hline 7503 & 918.0 & $12(4)$ & 0.87 & $9 \rightarrow 9$ & $21^{+} \rightarrow 19^{+}$ & E2 \\
\hline 7717 & 1004.9 & $41(10)$ & 1.08 & $1 \rightarrow 1$ & $22^{+} \rightarrow 20^{+}$ & E2 \\
\hline 7865 & 966.9 & - & 1.16 & $12 \rightarrow 12$ & $\left(21^{+}\right) \rightarrow\left(19^{+}\right)$ & E2 \\
\hline 7878 & 1007.4 & - & - & $5 \rightarrow 5$ & $21^{-} \rightarrow 19^{-}$ & E2 \\
\hline 7984 & 983.7 & $18(4)$ & 1.09 & $2 \rightarrow 2$ & $22^{+} \rightarrow 20^{+}$ & $\mathrm{E} 2$ \\
\hline 7984 & 1272.3 & - & - & $2 \rightarrow 1$ & $22^{+} \rightarrow 20^{+}$ & E2 \\
\hline 8101 & 1016.8 & $11(5)$ & 1.08 & $10 \rightarrow 10$ & $22^{+} \rightarrow 20^{+}$ & E2 \\
\hline 8101 & 1389.0 & - & - & $10 \rightarrow 1$ & $22^{+} \rightarrow 20^{+}$ & E2 \\
\hline 8263 & 1034.4 & $11(5)$ & 0.91 & $4 \rightarrow 4$ & $22^{-} \rightarrow 20^{-}$ & E2 \\
\hline 8371 & 1006.3 & - & 1.06 & $13 \rightarrow 13$ & $\left(22^{+}\right) \rightarrow\left(20^{+}\right)$ & E2 \\
\hline 8411 & 1044.0 & - & - & $6 \rightarrow 6$ & $22^{-} \rightarrow 20^{-}$ & E2 \\
\hline 8486 & 769.2 & - & - & $9 \rightarrow 1$ & $23^{+} \rightarrow 22^{+}$ & M1 \\
\hline 8486 & 983.4 & $10(4)$ & 1.11 & $9 \rightarrow 9$ & $23^{+} \rightarrow 21^{+}$ & E2 \\
\hline 8512 & 1009.7 & $14(4)$ & 0.95 & $3 \rightarrow 3$ & $23^{-} \rightarrow 21^{-}$ & E2 \\
\hline 8795 & 1077.1 & $37(9)$ & 1.22 & $1 \rightarrow 1$ & $24^{+} \rightarrow 22^{+}$ & E2 \\
\hline 8905 & 1040.6 & - & 1.22 & $12 \rightarrow 12$ & $\left(23^{+}\right) \rightarrow\left(21^{+}\right)$ & E2 \\
\hline 8913 & 1034.0 & - & - & $5 \rightarrow 5$ & $\left.23^{-} \rightarrow 21^{-}\right)$ & E2 \\
\hline 9054 & 1069.2 & $12(3)$ & 0.90 & $2 \rightarrow 2$ & $24^{+} \rightarrow 22^{+}$ & E2 \\
\hline 9179 & 1078.6 & $10(5)$ & 0.84 & $10 \rightarrow 10$ & $24^{+} \rightarrow 22^{+}$ & E2 \\
\hline 9179 & 1462.3 & - & - & $10 \rightarrow 1$ & $24^{+} \rightarrow 22^{+}$ & E2 \\
\hline
\end{tabular}


TABLE I: (continued)

Excitation Energy ${ }^{a}$ Intensity $^{b} R_{D C O}^{c}$ Band $_{i} \rightarrow$ Band $_{f}$ Assignment Multipolarity $E_{i}(\mathrm{keV}) \quad E_{\gamma}(\mathrm{keV}) \quad I_{\gamma} \quad$ ratio $\quad J_{i}^{\pi} \rightarrow J_{f}^{\pi}$

\begin{tabular}{ccccccc}
\hline \hline 9381 & 1117.6 & $5(1)$ & 0.89 & $4 \rightarrow 4$ & $24^{-} \rightarrow 22^{-}$ & E2 \\
9430 & 1058.5 & - & 1.25 & $13 \rightarrow 13$ & $\left(24^{+}\right) \rightarrow\left(22^{+}\right)$ & E2 \\
9528 & 1117.1 & - & - & $6 \rightarrow 6$ & $\left(24^{-}\right) \rightarrow\left(22^{-}\right)$ & E2 \\
9566 & 1078.2 & $6(2)$ & 1.25 & $9 \rightarrow 9$ & $25^{+} \rightarrow 23^{+}$ & E2 \\
9613 & 1100.1 & $8(2)$ & 1.37 & $3 \rightarrow 3$ & $25^{-} \rightarrow 23^{-}$ & E2 \\
9918 & 1124.1 & - & - & $11 \rightarrow 1$ & $(25) \rightarrow 24^{+}$ & - \\
9951 & 1154.5 & $15(6)$ & 1.11 & $1 \rightarrow 1$ & $26^{+} \rightarrow 24^{+}$ & E2 \\
9976 & 1070.6 & - & 1.07 & $12 \rightarrow 12$ & $\left(25^{+}\right) \rightarrow\left(23^{+}\right)$ & E2 \\
9984 & 1070.7 & - & - & $5 \rightarrow 5$ & $\left(25^{-}\right) \rightarrow\left(23^{-}\right)$ & E2 \\
10223 & 1168.6 & $10(3)$ & 0.97 & $2 \rightarrow 2$ & $26^{+} \rightarrow 24^{+}$ & E2 \\
10312 & 1131.4 & $5(2)$ & 1.16 & $10 \rightarrow 10$ & $26^{+} \rightarrow 24^{+}$ & E2 \\
10312 & 1516.9 & - & - & $10 \rightarrow 1$ & $26^{+} \rightarrow 24^{+}$ & E2 \\
10521 & 1091.9 & - & 1.12 & $13 \rightarrow 13$ & $\left(26^{+}\right) \rightarrow\left(24^{+}\right)$ & E2 \\
10563 & 1181.2 & $4(1)$ & - & $4 \rightarrow 4$ & $26^{-} \rightarrow 24^{-}$ & E2 \\
10707 & 1178.4 & - & - & $6 \rightarrow 6$ & $\left(26^{-}\right) \rightarrow\left(24^{-}\right)$ & E2 \\
10751 & 1184.8 & $2(2)$ & - & $9 \rightarrow 9$ & $27^{+} \rightarrow 25^{+}$ & E2 \\
10814 & 1201.5 & $6(2)$ & 1.29 & $3 \rightarrow 3$ & $27^{-} \rightarrow 25^{-}$ & E2 \\
11029 & 1151.3 & - & - & $11 \rightarrow 11$ & $(27) \rightarrow(25)$ & E2 \\
11079 & 1102.4 & - & 1.13 & $12 \rightarrow 12$ & $\left(27^{+}\right) \rightarrow\left(25^{+}\right)$ & E2 \\
11118 & 1505.0 & - & - & $3 b \rightarrow 3$ & $(26) \rightarrow 25^{-}$ & - \\
11183 & 1231.2 & $11(4)$ & 0.86 & $1 \rightarrow 1$ & $28^{+} \rightarrow 26^{+}$ & E2 \\
11475 & 1251.8 & $6.3(3.8)$ & 0.97 & $2 \rightarrow 2$ & $28^{+} \rightarrow 26^{+}$ & E2 \\
11526 & 1214.2 & $3(2)$ & 0.91 & $10 \rightarrow 10$ & $28^{+} \rightarrow 26^{+}$ & E2 \\
\hline & 1129.3 & - & 1.21 & $13 \rightarrow 13$ & $\left(28^{+}\right) \rightarrow\left(26^{+}\right)$ & E2 \\
\hline
\end{tabular}


TABLE I: (continued)

Excitation Energy $^{a}$ Intensity $^{b} R_{D C O}^{c}$ Band $_{i} \rightarrow$ Band $_{f}$ Assignment Multipolarity $E_{i}(\mathrm{keV}) \quad E_{\gamma}(\mathrm{keV}) \quad I_{\gamma} \quad$ ratio $\quad J_{i}^{\pi} \rightarrow J_{f}^{\pi}$

\begin{tabular}{ccccccc}
\hline \hline 11755 & 1192.0 & $2(1)$ & - & $4 \rightarrow 4$ & $28^{-} \rightarrow 26^{-}$ & E2 \\
12035 & 1282.9 & - & - & $9 \rightarrow 9$ & $29^{+} \rightarrow 27^{+}$ & E2 \\
12118 & 1304.4 & $5(1)$ & 1.02 & $3 \rightarrow 3$ & $29^{-} \rightarrow 27^{-}$ & E2 \\
12246 & 1164.9 & - & - & $12 \rightarrow 12$ & $\left(29^{+}\right) \rightarrow\left(27^{+}\right)$ & E2 \\
12290 & 1221.2 & - & - & $11 \rightarrow 11$ & $(29) \rightarrow(27)$ & E2 \\
12492 & 1309.6 & $10(3)$ & 1.27 & $1 \rightarrow 1$ & $30^{+} \rightarrow 28^{+}$ & E2 \\
12738 & 1261.2 & $4(1)$ & 0.93 & $2 \rightarrow 2$ & $30^{+} \rightarrow 28^{+}$ & E2 \\
12826 & 1297.9 & - & - & $10 \rightarrow 10$ & $30^{+} \rightarrow 28^{+}$ & E2 \\
12862 & 1210.9 & - & - & $13 \rightarrow 13$ & $\left(30^{+}\right) \rightarrow\left(28^{+}\right)$ & E2 \\
12961 & 1206.5 & - & - & $4 \rightarrow 4$ & $\left(30^{-}\right) \rightarrow\left(28^{-}\right)$ & E2 \\
13350 & 1232.0 & - & $0.8^{e}$ & $14 \rightarrow 3$ & $(30) \rightarrow 29^{-}$ & - \\
13413 & 1377.0 & - & - & $9 \rightarrow 9$ & $31^{+} \rightarrow 29^{+}$ & E2 \\
13498 & 1249.5 & - & - & $12 \rightarrow 12$ & $\left(31^{+}\right) \rightarrow\left(29^{+}\right)$ & E2 \\
13519 & 1400.8 & $4(2)$ & 1.18 & $3 \rightarrow 3$ & $31^{-} \rightarrow 29^{-}$ & E2 \\
13592 & 1301.4 & - & - & $11 \rightarrow 11$ & $(31) \rightarrow(29)$ & E2 \\
13881 & 1388.6 & $9(3)$ & 1.22 & $1 \rightarrow 1$ & $32^{+} \rightarrow 30^{+}$ & E2 \\
14063 & 1324.5 & $3.6(2.9)$ & - & $2 \rightarrow 2$ & $32^{+} \rightarrow 30^{+}$ & E2 \\
14185 & 1224.3 & - & - & $4 \rightarrow 4$ & $32^{-} \rightarrow 30^{-}$ & E2 \\
14196 & 1370.1 & - & - & $10 \rightarrow 10$ & $32^{+} \rightarrow 30^{+}$ & E2 \\
14757 & 1238.0 & - & $0.94^{e}$ & $14 \rightarrow 3$ & $(32) \rightarrow 31^{-}$ & - \\
14757 & 1406.9 & - & - & $14 \rightarrow 14$ & $(32) \rightarrow(30)$ & E2 \\
14838 & 1340.6 & - & - & $12 \rightarrow 12$ & $\left(33^{+}\right) \rightarrow\left(31^{+}\right)$ & E2 \\
14887 & 1474.7 & - & - & $9 \rightarrow 9$ & $33^{+} \rightarrow 31^{+}$ & E2 \\
\hline & 1389.1 & - & - & $11 \rightarrow 11$ & $(33) \rightarrow(31)$ & E2 \\
\hline
\end{tabular}


TABLE I: (continued)

Excitation Energy $^{a}$ Intensity $^{b} R_{D C O}^{c}$ Band $_{i} \rightarrow$ Band $_{f}$ Assignment Multipolarity $E_{i}(\mathrm{keV}) \quad E_{\gamma}(\mathrm{keV}) \quad I_{\gamma} \quad$ ratio $\quad J_{i}^{\pi} \rightarrow J_{f}^{\pi}$

\begin{tabular}{ccccccc}
\hline \hline 15007 & 1486.5 & - & - & $3 \rightarrow 3$ & $33^{-} \rightarrow 31^{-}$ & E2 \\
15336 & 1454.8 & $6(2)$ & $1.38^{d}$ & $1 \rightarrow 1$ & $34^{+} \rightarrow 32^{+}$ & E2 \\
15465 & 1401.6 & - & - & $2 \rightarrow 2$ & $34^{+} \rightarrow 32^{+}$ & E2 \\
15476 & 1290.9 & - & - & $4 \rightarrow 4$ & $\left(34^{-}\right) \rightarrow 32^{-}$ & E2 \\
15624 & 1427.5 & - & - & $10 \rightarrow 10$ & $34^{+} \rightarrow 32^{+}$ & E2 \\
16031 & 1274.0 & - & $1.42^{e}$ & $14 \rightarrow 14$ & $(34) \rightarrow(32)$ & E2 \\
16286 & 1448.0 & - & - & $12 \rightarrow 12$ & $\left(35^{+}\right) \rightarrow\left(33^{+}\right)$ & E2 \\
16434 & 1544.2 & - & - & $9 \rightarrow 9$ & $\left(35^{+}\right) \rightarrow 33^{+}$ & E2 \\
16463 & 1481.8 & - & - & $11 \rightarrow 11$ & $(35) \rightarrow(33)$ & E2 \\
16780 & 1444.3 & $5(2)$ & $1.41^{d}$ & $1 \rightarrow 1$ & $36^{+} \rightarrow 34^{+}$ & E2 \\
16918 & 1583.4 & - & - & $1 c \rightarrow 1$ & $\left(36^{+}\right) \rightarrow 34^{+}$ & E2 \\
16949 & 1484.3 & - & - & $2 \rightarrow 2$ & $36^{+} \rightarrow 34^{+}$ & E2 \\
17117 & 1493.3 & - & - & $10 \rightarrow 10$ & $\left(36^{+}\right) \rightarrow 34^{+}$ & E2 \\
17437 & 1406.0 & - & - & $14 \rightarrow 14$ & $(36) \rightarrow(34)$ & E2 \\
18042 & 1579.7 & - & - & $11 \rightarrow 11$ & $(37) \rightarrow(35)$ & E2 \\
18055 & 1619.6 & - & - & $9 \rightarrow 9$ & $\left(37^{+}\right) \rightarrow\left(35^{+}\right)$ & E2 \\
18075 & 1294.4 & - & - & $1 a \rightarrow 1$ & $\left(38^{+}\right) \rightarrow 36^{+}$ & E2 \\
18149 & 1368.4 & - & - & $1 b \rightarrow 1$ & $\left(38^{+}\right) \rightarrow 36^{+}$ & E2 \\
18529 & 1610.9 & - & - & $1 c \rightarrow 1 c$ & $\left(38^{+}\right) \rightarrow\left(36^{+}\right)$ & E2 \\
18656 & 1537.7 & - & - & $10 \rightarrow 10$ & $\left(38^{+}\right) \rightarrow\left(36^{+}\right)$ & E2 \\
18911 & 1474.0 & - & - & $14 \rightarrow 14$ & $(38) \rightarrow(36)$ & E2 \\
19722 & 1679.7 & - & - & $11 \rightarrow 11$ & $(39) \rightarrow(37)$ & E2 \\
20485 & 1574.0 & - & - & $14 \rightarrow 14$ & $(40) \rightarrow(38)$ & E2 \\
\hline & 1780.9 & - & - & $11 \rightarrow 11$ & $(41) \rightarrow(39)$ & E2 \\
\hline
\end{tabular}


TABLE I: (continued)

\begin{tabular}{ccccccc} 
Excitation & Energy $^{a}$ & Intensity & $R_{D C O}^{c}$ & Band $_{i} \rightarrow$ Band $_{f}$ & Assignment & Multipolarity \\
$E_{i}(\mathrm{keV})$ & $E_{\gamma}(\mathrm{keV})$ & $I_{\gamma}$ & ratio & & $J_{i}^{\pi} \rightarrow J_{f}^{\pi}$ & \\
& & & & & \\
\hline \hline 22152 & 1666.8 & - & - & $14 \rightarrow 14$ & $(42) \rightarrow(40)$ & $\mathrm{E} 2$ \\
23386 & 1883.2 & - & - & $11 \rightarrow 11$ & $(43) \rightarrow(41)$ & $\mathrm{E} 2$ \\
25372 & 1986.3 & - & - & $11 \rightarrow 11$ & $(45) \rightarrow(43)$ & $\mathrm{E} 2$ \\
\hline \hline
\end{tabular}

${ }^{a}$ Uncertainties of $\gamma$-ray energies are between 0.1 and $0.6 \mathrm{keV}$ depending on their intensity.

${ }^{b}$ Intensities are normalized to the $576.5 \mathrm{keV}$ transition with $\mathrm{I}_{\gamma}=500$.

${ }^{c}$ Uncertainties in the DCO rations are between 0.03 and 0.2.

${ }^{d}$ Ratio obtained from angular distribution matrices.

${ }^{e}$ Uncertainty $>50 \%$ due to low statistics. 


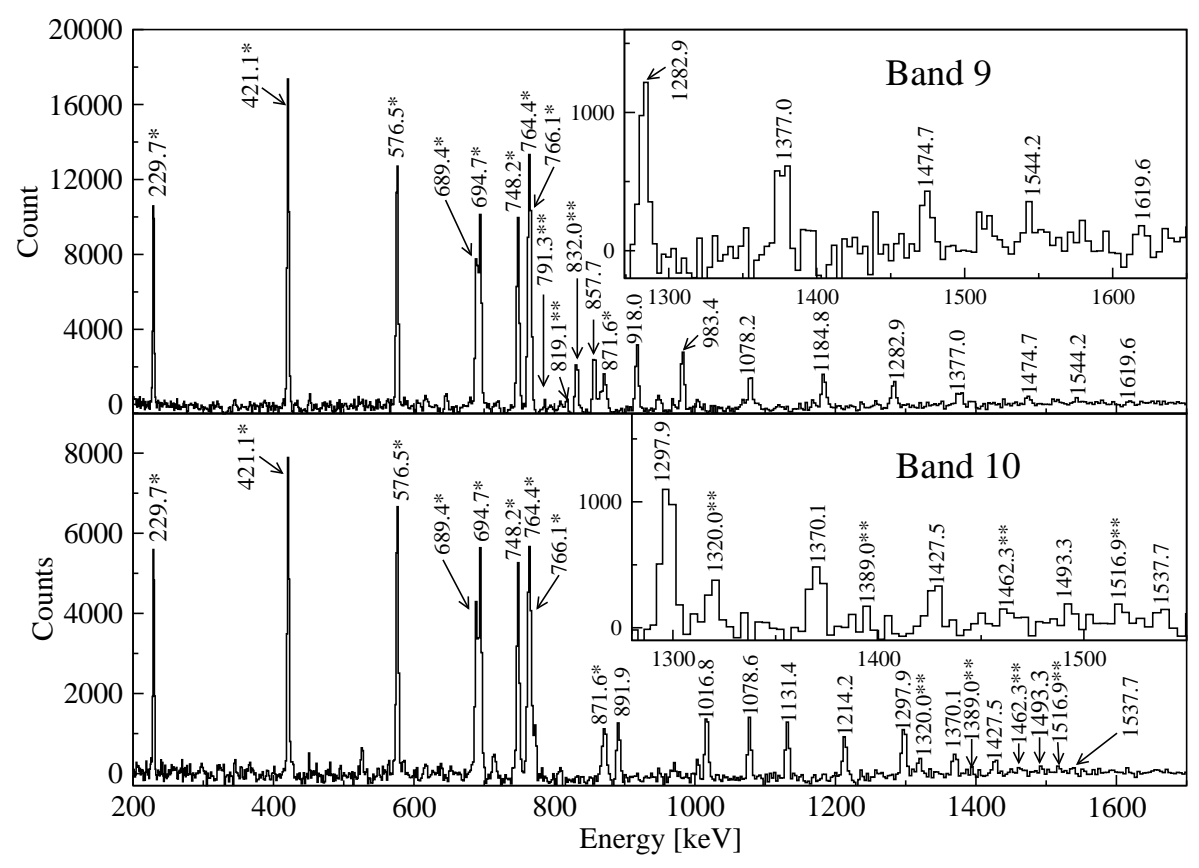

FIG. 3: Sum of triple-gated $\gamma$-ray coincidence spectra of Band 9 (upper panel) and Band 10 (lower panel). The spectrum of Band 9 was produced by setting one gate on transitions (229.7 to 766.1 $\mathrm{keV}$ ) of Band 1 and two gates on transitions (832 to $1619.6 \mathrm{keV}$ ) of Band 9. The spectrum of Band 10 was obtained with one gate on the same transitions of Band 1 and two gates on transitions (891.9 to $1537.7 \mathrm{keV}$ ) of Band 10. The single asterisks mark peaks of Band 1. The $\gamma$ rays marked by double asterisks are the linking transitions from Bands 9 and 10, respectively, to Band 1.

Bands 3 to 8 were also known from previous work $[12,18]$. We confirm these sequences and place three to four transitions on top of the previously known levels of Bands 3, 5 and 6 . The low-spin part of the coupled Bands 5 and 6 was rearranged. Two close-lying $7^{-}$states, at 2647 and $2691 \mathrm{keV}$, respectively, were previously known [12]. The $2647 \mathrm{keV}$ level was assigned as member of the $\alpha=1$ signature branch whereas the $2691 \mathrm{keV}$ state was suggested not to belong to the band. Analyzing triple coincidences, we found a $419.2 \mathrm{keV}$ transition from the $9^{-}$band member to the $7^{-}$level at $2691 \mathrm{keV}$ which was previously not detected, probably because it lies so close in energy to the strong $421.1 \mathrm{keV} 4^{+} \rightarrow 2^{+}$transition. We rearrange the $7^{-}$states as shown in the level scheme of Fig. 1. In this way the coupled bands continue with $\Delta I=1$ and $\Delta I=2$ transitions down to the $5^{-}$state. The fairly strong transition of $462.0 \mathrm{keV}$ to the $7^{-}$state at $2647 \mathrm{keV}$ may be explained by mixing of the $7^{-}$ levels.

Band 4 is extended by seven transitons. Bands 7 and 8 are weakly populated and could 
not be extended to higher spin. DCO ratios measured for some of the in-band transitions as well as decay-out transitions are in accordance with the previous spin assignments [12]. For some high-spin transitions observed in this work we could not determine the multipolarity. However, E2 multipolaritiy has been assumed for the in-band transitions due to the regularity of the bands.

The two sequences of $\gamma$ rays labeled Band 9 and 10 have been observed for the first time in our work. Both bands decay into levels of Band 1. Gamma-ray coincidence spectra for the two bands are shown in Fig. 3. The $832.0 \mathrm{keV}$ transition, which we place at the bottom of Band 9, was observed already by Pilotte et al. [12]. The linear polarization asymmetry of this transition is small and negative which confirms the previously suggested mixed M1/E2 multipolarity for this transition which feeds into the $16^{+}$state of Band 1. For an electric dipole one would expect no mixing and, thus, a positive asymmetry. This leads to $I^{\pi}=17^{+}$ for the lowest level of Band 9.

Band 10 decays into Band 1 via several transitions between 1299 and $1517 \mathrm{keV}$. Of these only the $1319.7 \mathrm{keV}$ transition is clean and strong enough to allow the determination of the angular correlation ratio. This ratio is compatible with stretched quadrupole, probably E2, multipolarity. Thus, we assign even spins and positive parity to Band 10. Only the in-band transitions near the bottom of Bands 9 and 10 are sufficiently strong for a determination of the angular correlation ratios. They are consistent with stretched quadrupole, probably E2, transitions.

The three new bands, Bands 11, 12 and 13, are only tentatively connected to lower-lying levels and their excitation energies, spins and parities remain uncertain. Gamma-ray coincidence spectra of these bands are displayed in Figs. 4 and 5, respectively. Band 11 is a cascade of eleven transitions, probably of E2 multipolarity, which decays into Band 1. Transitions of Band 1 are observed in coincidence with the new band up to the $24^{+}$level. The transitions of Band 11 show an energy difference which is smoothly increasing from 71 to $105 \mathrm{keV}$; only the difference between the 1124.1 and $1151.3 \mathrm{keV}$ transitions is much lower. Therefore, the $1124.1 \mathrm{kev} \gamma$ ray may be a link between the two bands. Other weak $\gamma$-ray lines observed in the coincidence spectra, see Fig. 4, with energies of 1095, 1453, 1516, 1833 and $1914 \mathrm{keV}$ might also be candidates for inter-band transitions.

The two other new bands, Bands 12 and 13, are connected by weak inter-band transitions in the low-spin region and, thus, probably form a pair of signature-partner bands. They decay into Bands 1, 2 and 7. Of the linking transitions shown in the level scheme, Fig. 1, only the $1407 \mathrm{keV}$ line is firmly established. Due to the low intensity its DCO ratio has a large uncertainty and the multipolarity could not be unambigously determined. Hence, the spin assignment to the bands is tentative. The other transitions are weak and only tentatively 


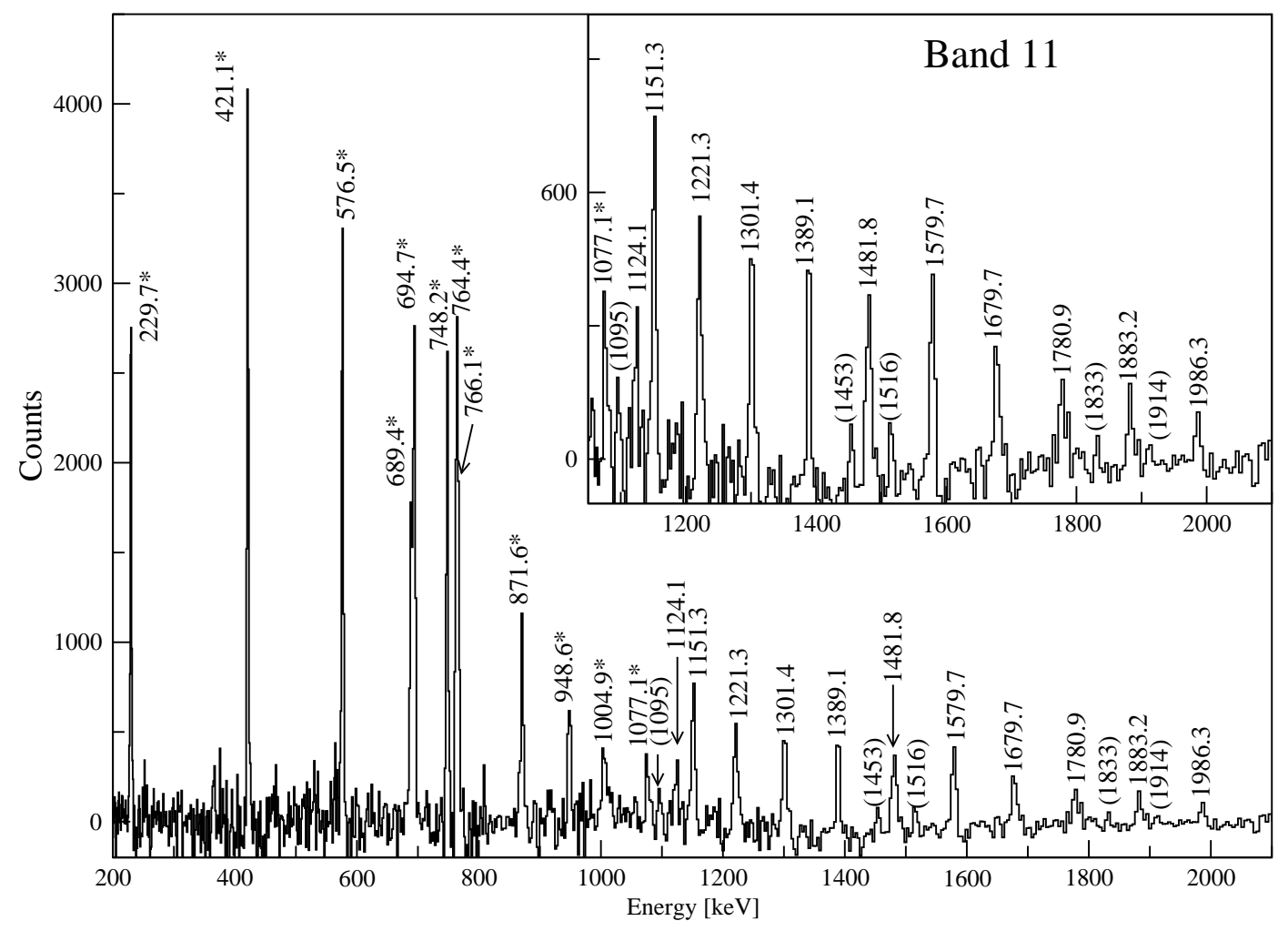

FIG. 4: Summed triple-gated coincidence spectrum of Band 11. The spectrum was produced by setting one gate on transitions (229.7 to $1077 \mathrm{keV}$ ) of Band 1 and two gates on transitions (1124.1 to $1986.3 \mathrm{keV}$ ) of Band 11. The asterisks mark peaks of Band 1. The energies in parentheses correspond to transitions which are observed in coincidence but could not be placed in the level scheme.

assigned. Transitions of Band 1 up to the $10^{+}$and possibly up to the $14^{+}$state are observed in coincidence with Bands 12 and 13, see Fig. 5.

Band 14 has been observed for the first time in this work. It decays into Band 3. Due to the low intensities of the linking transitions the DCO ratios have large uncertainties and their multipolarities could not be reliably determined. Since Band 14 feeds into the $I^{\pi}=$ $29^{-}$state of Band 3 we tentatively assign $I=30$ to the lowest level of the band. 


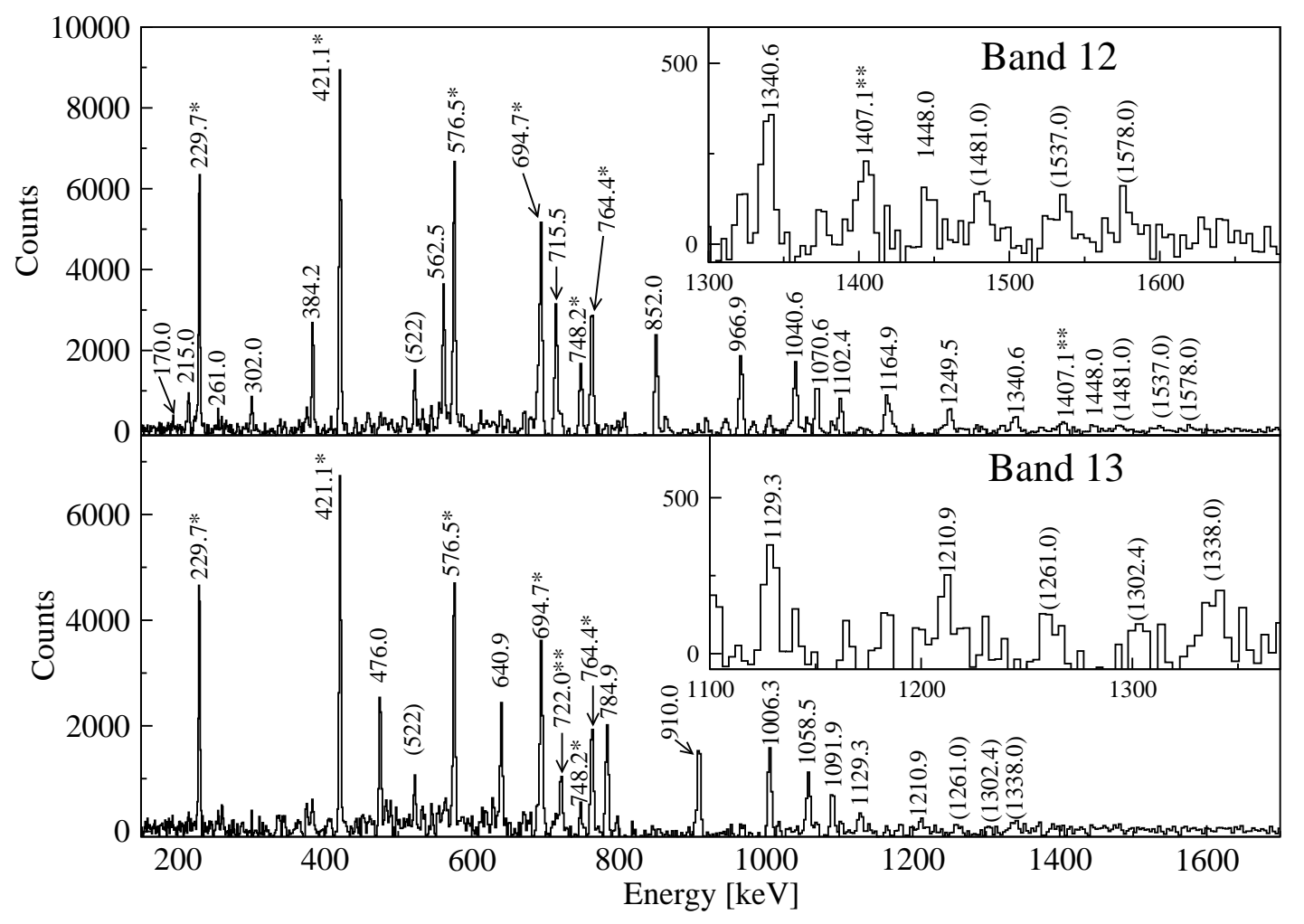

FIG. 5: Summed double-gated and triple-gated coincidence spectra of Bands 12 and 13. The spectrum of Band 12 (upper panel) was produced by setting one gate on transitions (229.7 to 764.4 $\mathrm{keV}$ ) of Band 1 and two gates on transitions (384.2 to $1448.0 \mathrm{keV}$ ) of Band 12. The spectrum of Band 13 (lower panel) was obtained by setting one gate on the same transitions of Band 1 and two gates on transitions (476 to $1163 \mathrm{keV}$ ) of Band 13. The single asterisks mark peaks of Band 1. Double asterisks mark the decay-out transitions. The energies in parentheses correspond to transitions which are observed in coincidence but could not be placed in the level scheme.

\section{DISCUSSION}

In previous work $[12,18,32,33]$ configuration assignments have been made to the low- and medium-spin regions of Bands 1 to 8 of ${ }^{124} \mathrm{Ba}$. Arguments for the configuration assignments are the observed excitation energies, spins, parities, band-crossing frequencies and alignment gains. These quantities can be compared to those observed in neighboring nuclei as well 
as to cranked shell model (CSM) [34, 35] and cranked Nilsson-Strutinsky (CNS) [19, 36] calculations. For the coupled bands, signature splittings and $B(M 1) / B(E 2)$ ratios provide additional evidence for their configurations.

TABLE II: Shell model origin and quasiparticle labels for low-lying orbitals in ${ }^{124} \mathrm{Ba}$ [12].

\begin{tabular}{|c|c|c|c|c|}
\hline & Shell model states & Nilsson orbitals & $\alpha=+\frac{1}{2}$ & $\alpha=-\frac{1}{2}$ \\
\hline \multirow[t]{5}{*}{ Protons } & $\left(d_{5 / 2}, g_{7 / 2}\right)$ & {$[422] \frac{3}{2}^{+}$} & $a$ & $b$ \\
\hline & $\left(d_{5 / 2}, g_{7 / 2}\right)$ & {$[420] \frac{1}{2}^{+}$} & $c$ & $d$ \\
\hline & $g_{9 / 2}$ & {$[404] \frac{9}{2}^{+}$} & $a^{\prime}$ & $b^{\prime}$ \\
\hline & $h_{11 / 2}$ & {$[550] \frac{1}{2}^{-}$} & $f$ & $e$ \\
\hline & $h_{11 / 2}$ & {$[541] \frac{3}{2}^{-}$} & $h$ & $g$ \\
\hline \multirow[t]{5}{*}{ Neutrons } & $\left(s_{1 / 2}, d_{3 / 2}\right)$ & {$[411] \frac{1}{2}^{+}$} & $A$ & $B$ \\
\hline & $\left(d_{5 / 2}, g_{7 / 2}\right)$ & {$[402] \frac{5}{2}^{+}$} & $C$ & $D$ \\
\hline & $\left(d_{5 / 2}, g_{7 / 2}\right)$ & {$[413] \frac{5}{2}^{+}$} & $A^{\prime}$ & $B^{\prime}$ \\
\hline & $h_{11 / 2}$ & {$[523] \frac{7}{2}^{-}$} & $F$ & $E$ \\
\hline & $h_{11 / 2}$ & {$[532] \frac{5}{2}^{-}$} & $H$ & $G$ \\
\hline
\end{tabular}

In the discussion of the configurations of the observed bands we distinguish between the low- and high-spin regions. At low and medium spins pairing correlations are important and comparison of experiment with theory is usually made within the framework of the CSM. Here, we adopt the results of previous work [12] for Bands 1 to 8 and make suggestions for the configuration assignments for the new bands. The quasiparticle levels relevant in this mass region are given in Table II where we adopt the standard notation introduced with the CSM [34]. The Coriolis interaction mixes the original shell model states in the rotating nucleus. Nevertheless, we include the shell model and Nilsson configurations for convenience. For the higher-spin states, typically for states with $I>20$, calculations have been performed using the configuration-dependent CNS formalism without pairing $[19,36]$. The present calculations are similar to those presented for ${ }^{123} \mathrm{Cs}$ in ref. [21]. Results of these calculations are compared with experimental excitation energies in Figs. 6 and 7 for the positive- and negative-parity bands, respectively. 


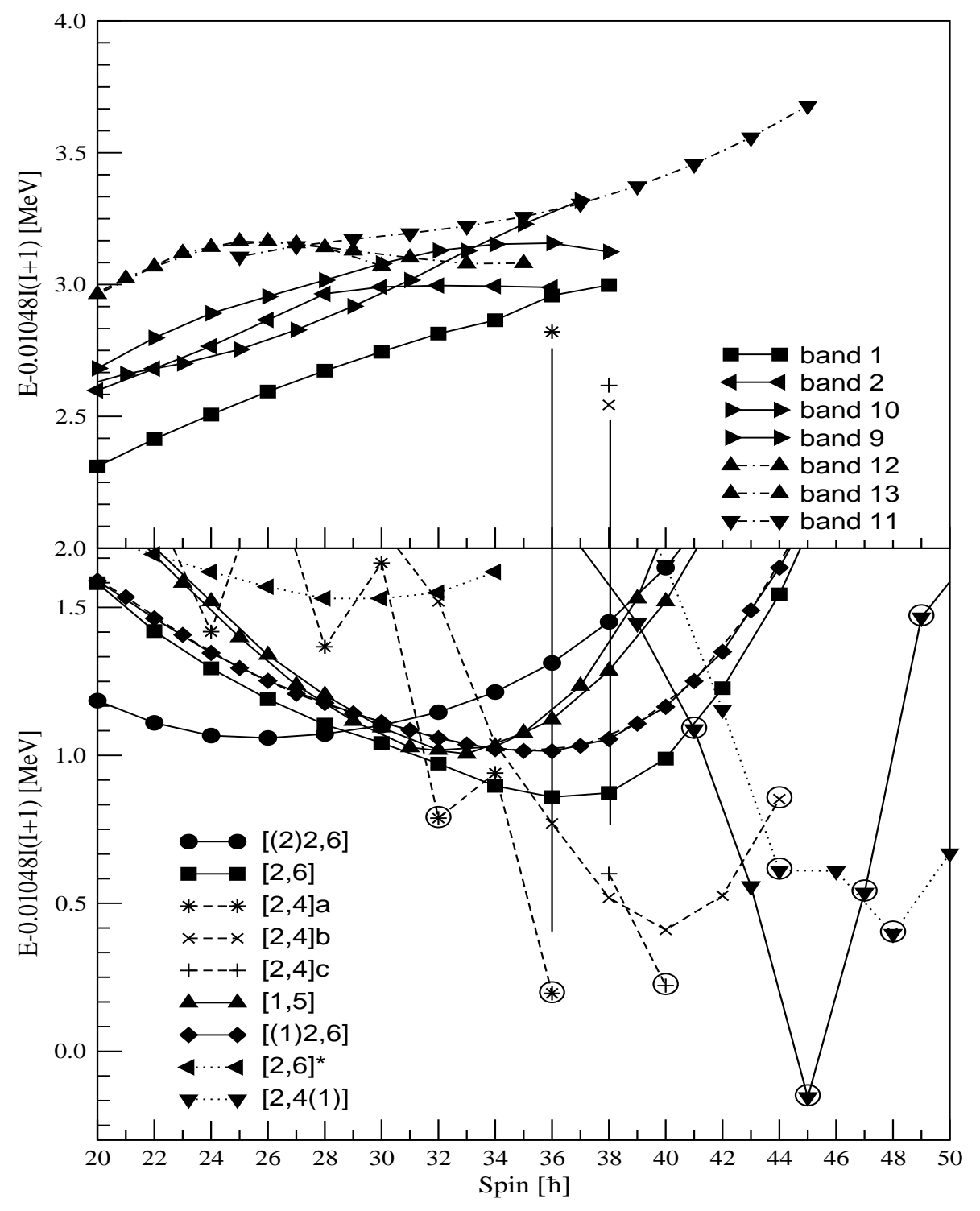

FIG. 6: Experimental excitation energies relative to a rigid-rotor reference as a function of spin for the positive-parity bands in ${ }^{124} \mathrm{Ba}$ (upper panel) compared to energies calculated within the framework of the CNS model (lower panel)

The CNS configurations are labeled by the number of particles in the different high- $j$ intruder orbitals outside a closed core. However, it should be noted that in this formalism no separation into core and valence particles is made and all orbitals up to $N=8$ are treated on an equal footing. In the case of ${ }^{124} \mathrm{Ba}$ it is natural to choose ${ }^{114} \mathrm{Sn}$ with $Z=50$ and $N=64$ as the core. The configurations may then be identified by the number of proton holes in the $g_{9 / 2}$ subshell, the number of protons in $h_{11 / 2}$ orbitals and the number of neutrons in $h_{11 / 2}$ and $i_{13 / 2}$ orbitals. The notation for labeling the configurations is given in the form $\left[\left(p_{0}\right) p_{1}, n_{0}\left(n_{1}\right)\right]$, where $p_{0}$ is the number of proton holes in $g_{9 / 2}$ orbitals (omitted when 


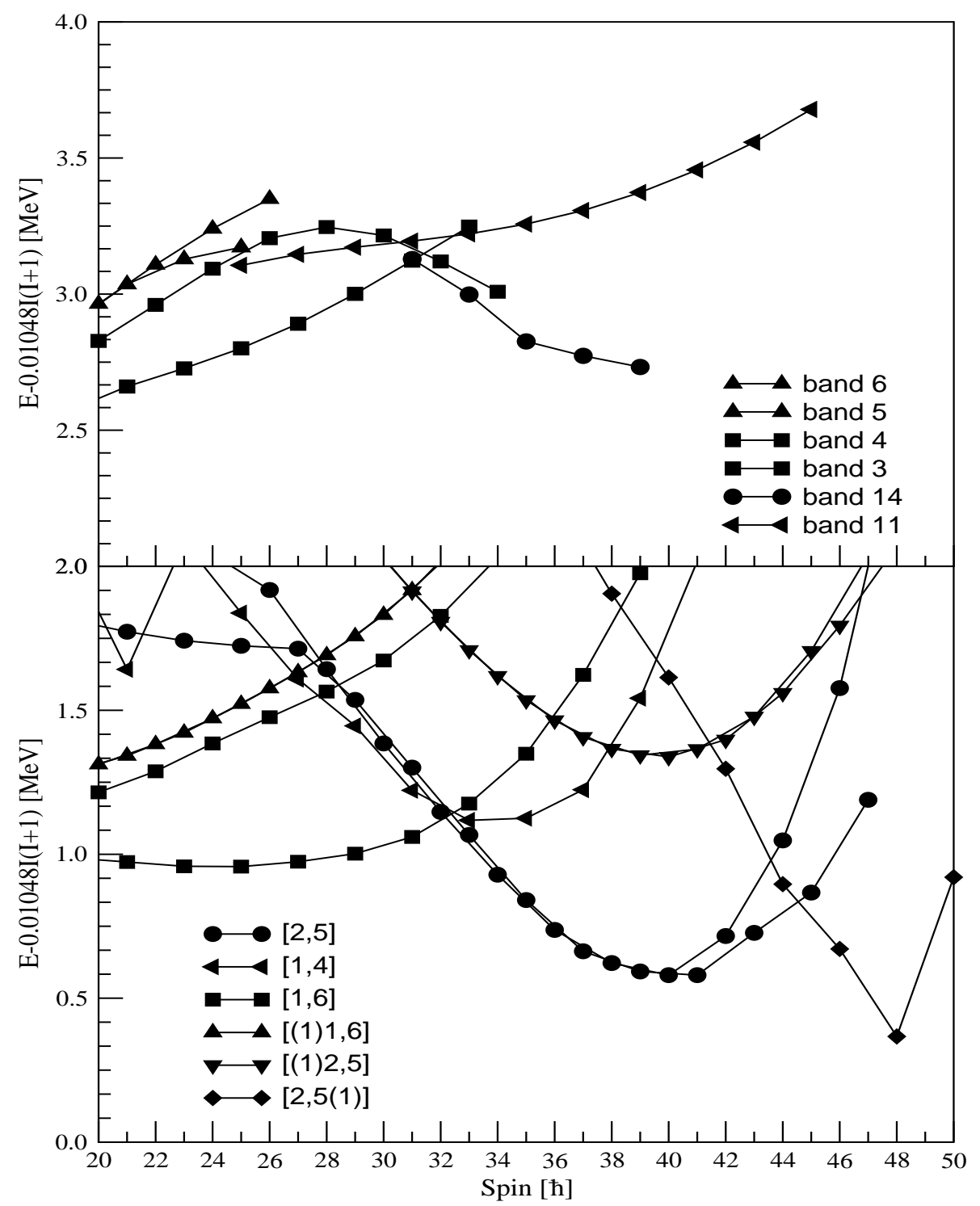

FIG. 7: Experimental excitation energies relative to a rigid-rotor reference as a function of spin for negative-parity bands in ${ }^{124} \mathrm{Ba}$ (upper panel) compared to energies calculated within the framework of the CNS model (lower panel)

$\left.p_{0}=0\right), p_{1}$ is the number of $h_{11 / 2}$ protons, $n_{0}$ is the number of neutrons in the $h_{11 / 2}$ subshell and $n_{1}$ the number of neutrons in $i_{13 / 2}$ orbitals (omitted when $n_{1}=0$ ). The energy of each configuration is minimized at each spin in the deformation space $\left(\varepsilon_{2}, \varepsilon_{4}, \gamma\right)$, which allows the development of collectivity to be treated as a function of spin.

In the CNS calculations we have used the parameters previously derived for the $A=130$ region [37]. As pairing is not included, the results should only be compared with experimental data at high spins where pairing is quenched. We note that there is a difference between 

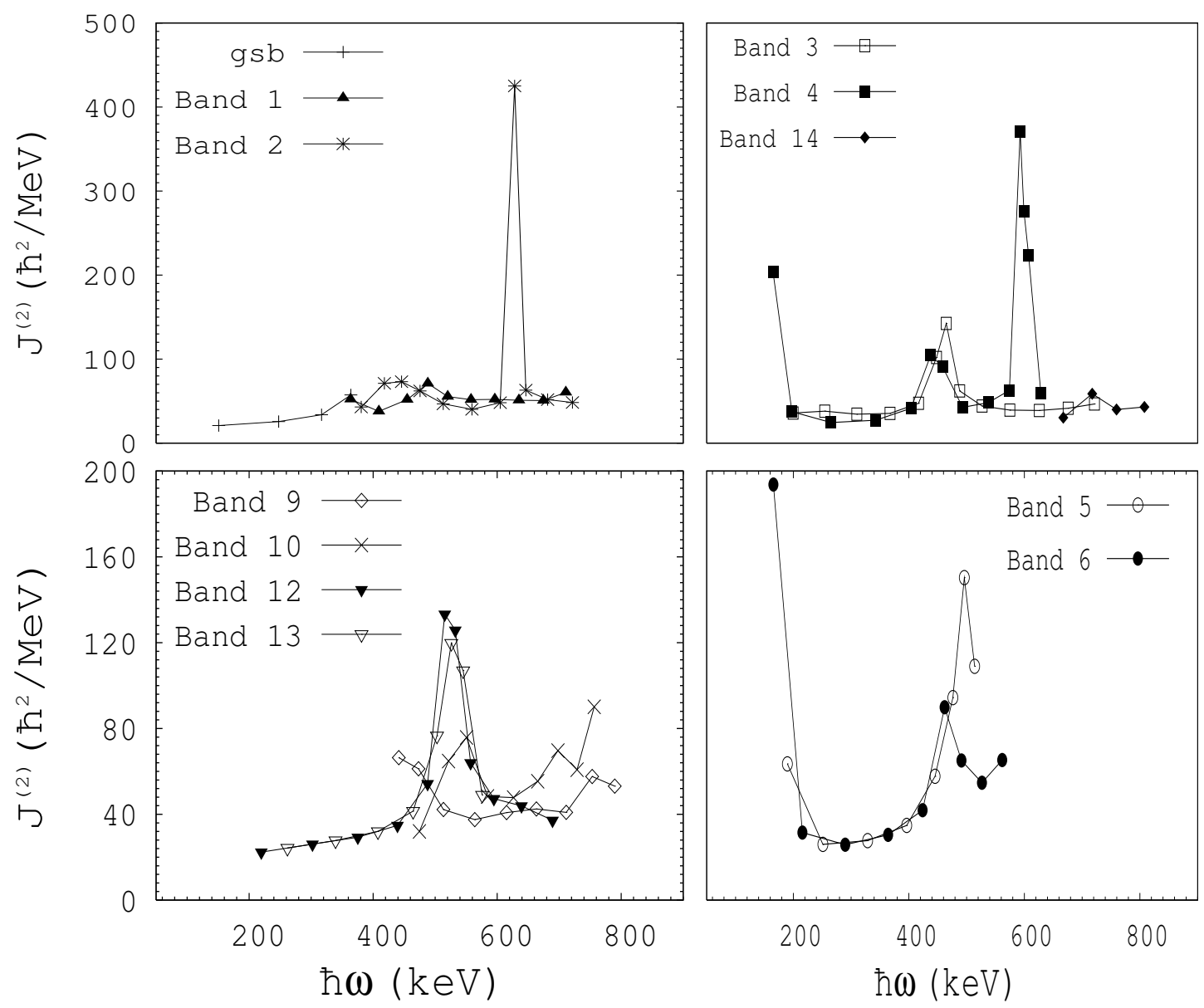

FIG. 8: Dynamic moment of inertia, $\mathcal{J}^{(2)}$, as a function of rotational frequency taken as $\hbar \omega=E_{\gamma} / 2$ for all observed bands.

the absolute values of the experimental and calculated level energies. The reason is that the experimental level energies are given relative to the ground-state energy while the reference of the calculated levels is the liquid drop energy at spin zero. Thus, the difference between the experimental and calculated energies depends essentially on the ground-state shell and pairing energy.

The experimental dynamic moments of inertia, aligned angular momenta and routhians for bands in ${ }^{124} \mathrm{Ba}$ are shown in Figs. 8, 9 and 10, respectively. A rigid-rotor reference with the parameters $\mathcal{J}_{\circ}=17 \hbar^{2} \mathrm{MeV}^{-1}$ and $\mathcal{J}_{1}=26 \hbar^{4} \mathrm{MeV}^{-3}$ has been used [38]. The experimental crossing frequencies and the alignment gains are listed in Table III. As can be seen in Fig. 9, up to medium frequencies the aligned angular momenta, $i_{x}$, increase roughly in two discrete steps, one at low frequencies, around $\hbar \omega=0.37 \mathrm{MeV}$, and another one at higher frequencies, around $\hbar \omega=0.45 \mathrm{MeV}$. The alignment gains may be attributed to the decoupling and alignment of quasiparticles of $h_{11 / 2}$ origin, either $h_{11 / 2}$ quasiprotons or $h_{11 / 2}$ 

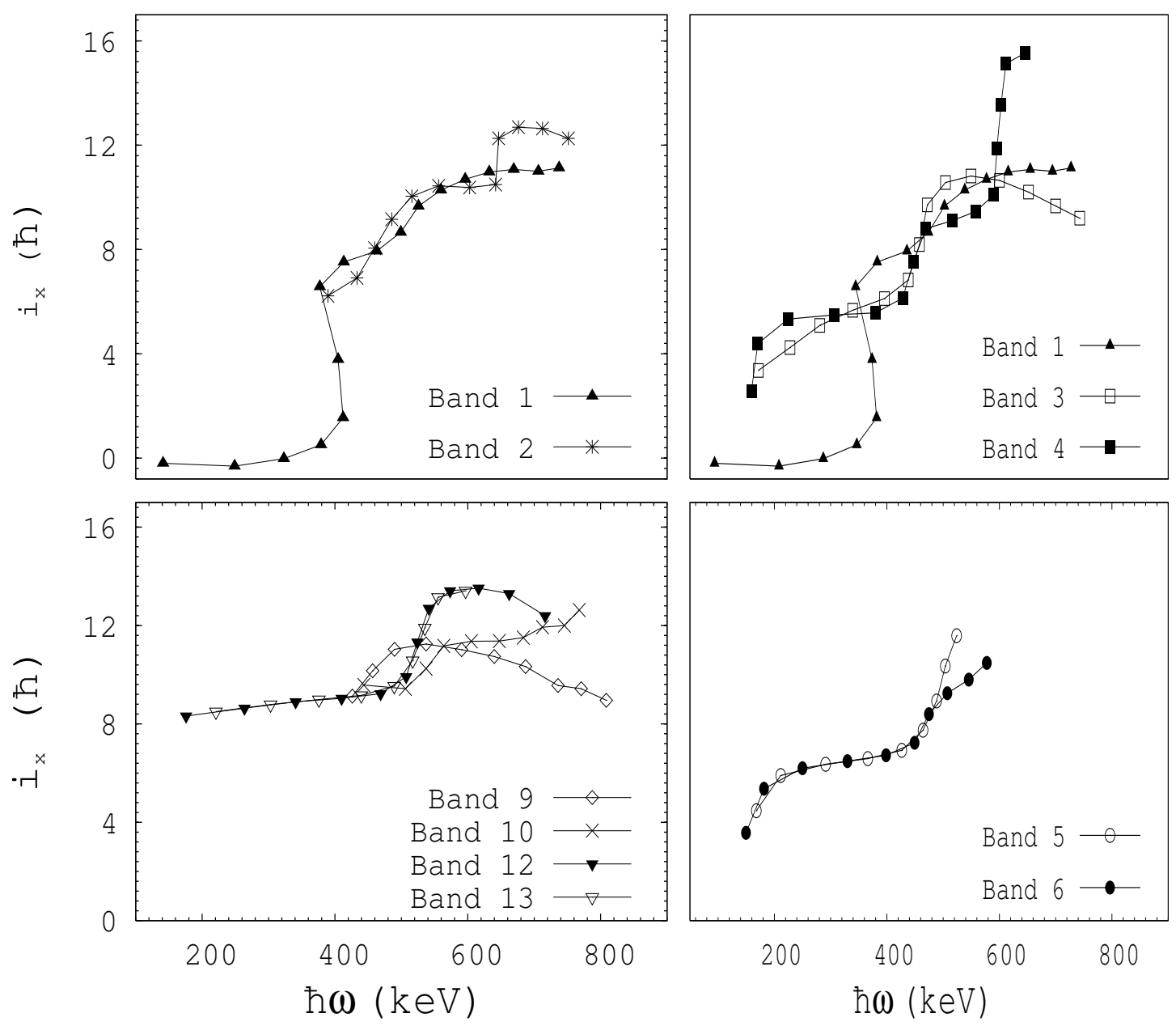

FIG. 9: Aligned angular momentum, $i_{x}$, as a function of rotational frequency. A reference core with Harris parameters $\mathcal{J}_{\circ}=17 \hbar^{2} \mathrm{MeV}^{-1}$ and $\mathcal{J}_{1}=26 \hbar^{4} \mathrm{MeV}^{-3}$ has been subtracted.

quasineutrons. The smaller differences in alignment between the various bands are then caused by different quasiparticles with lower angular momentum, $j$, in the configurations. Furthermore, deformation changes and differences in pairing may influence alignments. At the highest observed frequencies some of the bands show an additional increase in alignment or other irregularities. In the following we will discuss the configurations for the various bands.

\section{A. Bands 1 and 2}

Band 1 shows band crossings at rotational frequencies of 0.37 and $0.49 \mathrm{MeV} / \hbar$ with alignment gains of 8.2 and $3.3 \hbar$, respectively, see Fig. 9. The first alignment has been interpreted as 

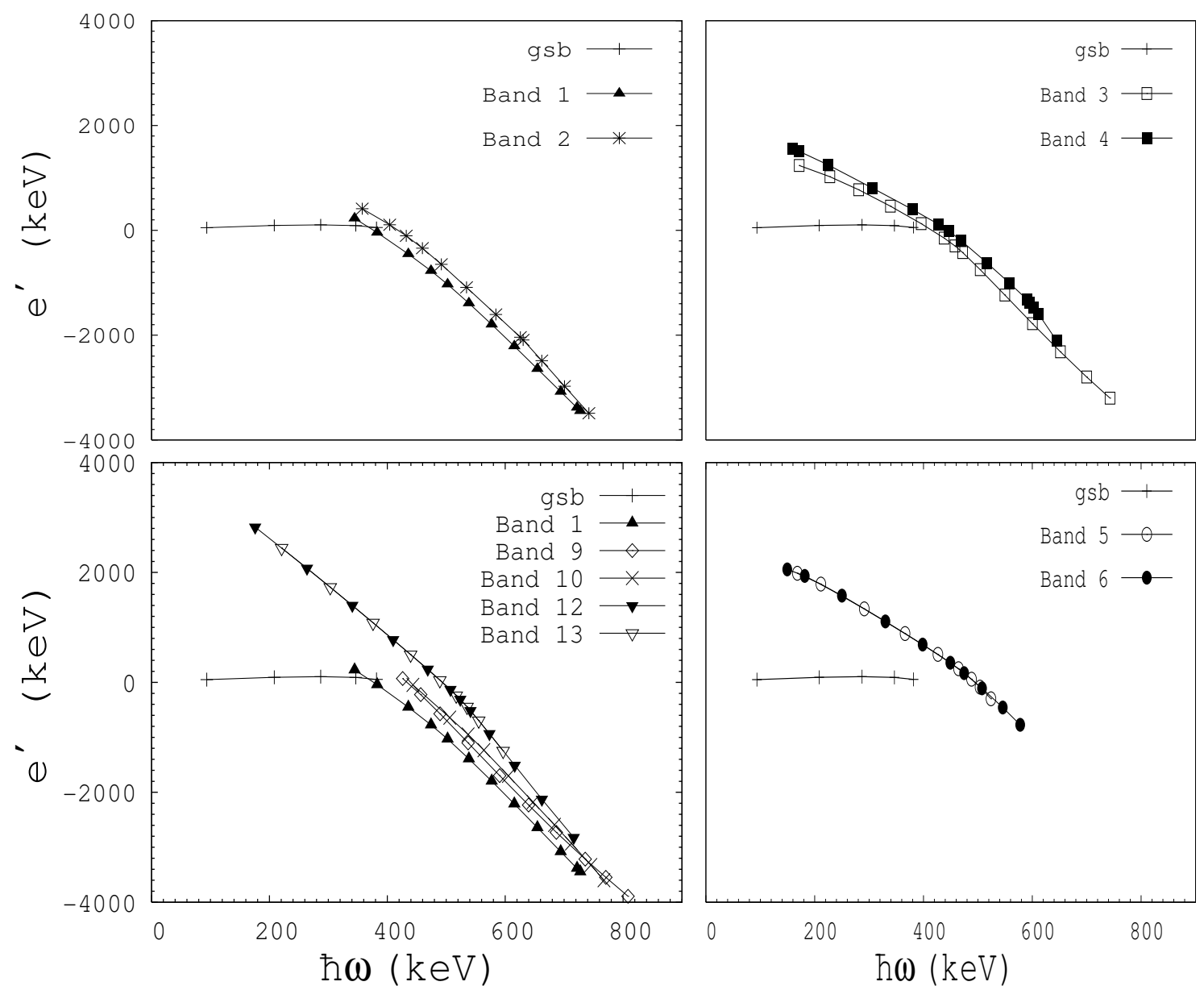

FIG. 10: Experimental routhians, $e^{\prime}$, as a function of rotational frequency. A reference core with Harris parameters $\mathcal{J}_{\circ}=17 \hbar^{2} \mathrm{MeV}^{-1}$ and $\mathcal{J}_{1}=26 \hbar^{4} \mathrm{MeV}^{-3}$ has been subtracted.

crossing of the ground band with the two-quasiproton $h_{11 / 2}$ band, ef, and the second gain in alignment was suggested to result from a decoupling of an $h_{11 / 2}$ neutron pair, EF [12]. Therefore, the configuration of Band 1 changes from the zero-quasiparticle ground-state band (gsb) to a two-quasiproton configuration, ef, and then to the two-quasiproton-twoquasineutron configuration, efEF.

The CNS calculations predict the $[2,6]\left(\pi h_{11 / 2}^{2} \otimes \nu h_{11 / 2}^{6}\right)$ configurations to be yrast above spin 28 until it is crossed by the steeply downsloping $[2,4]\left(\pi h_{11 / 2}^{2} \otimes \nu h_{11 / 2}^{4}\right)$ configuration, see Fig. 6. At lower spins, the CNS calculations predict the $[(2) 2,6]\left(\pi\left[\left(g_{9 / 2}^{-2}\right) h_{11 / 2}^{2}\right] \otimes \nu h_{11 / 2}^{6}\right)$ configuration below the $[2,6]$ structure. However, the calculated relative positions of the $[2,6]$ and $[(2) 2,6]$ configurations can depend on the Nilsson parameters and there is some uncertainty in their relative positions. Indeed, in the neighboring ${ }^{123} \mathrm{Cs}$ nucleus these calculations also predicted the $[(2) 2,6]$ below the $[2,6]$ configuration in that spin region, while the ob- 
TABLE III: Experimental crossing frequencies and aligned angular momenta in ${ }^{124} \mathrm{Ba}$.

\begin{tabular}{|c|c|c|c|c|c|c|c|c|c|c|}
\hline Band & $\begin{array}{c}\text { Before crossing } \\
\text { Config. }\end{array}$ & $\begin{array}{l}i_{x} \\
{[\hbar]}\end{array}$ & $\begin{array}{c}\hbar \omega_{c} \\
{[\mathrm{MeV}]}\end{array}$ & $\begin{array}{c}\Delta i_{x} \\
{[\hbar]}\end{array}$ & $\begin{array}{l}\text { Config. } \\
\text { above } 1^{s t} \\
\text { crossing }\end{array}$ & $\begin{array}{c}\hbar \omega_{c} \\
{[\mathrm{MeV}]}\end{array}$ & $\begin{array}{c}\Delta i_{x} \\
{[\hbar]}\end{array}$ & $\begin{array}{l}\text { Config. } \\
\text { above } 2^{\text {nd }} \\
\text { crossing }\end{array}$ & $\begin{array}{c}\hbar \omega_{c} \\
{[\mathrm{MeV}]}\end{array}$ & $\begin{array}{r}\Delta i_{x} \\
{[\hbar]}\end{array}$ \\
\hline 1 & $0-q p$ & 0 & 0.37 & 8.2 & ef & 0.49 & 3.3 & efEF & - & - \\
\hline 2 & $0-q p$ & 0 & 0.41 & 6.2 & $\mathrm{EF}$ & 0.44 & 4.4 & EFef & 0.63 & 2.5 \\
\hline 3 & $\mathrm{eb}$ & $\approx 5.1$ & 0.46 & 6.0 & $\mathrm{ebEF}$ & - & - & - & - & - \\
\hline 4 & ea & 5.4 & 0.44 & 4.0 & eaGH & 0.59 & $\approx 6.0$ & eaGHEF & - & - \\
\hline 5 & $\mathrm{eb}^{\prime}$ & 5.8 & 0.44 & $>6.1$ & $\mathrm{eb}^{\prime} \mathrm{EF}$ & - & - & - & - & - \\
\hline 6 & $\mathrm{ea}^{\prime}$ & 5.8 & 0.44 & $>5.8$ & $\mathrm{ea}^{\prime} \mathrm{EF}$ & - & - & - & - & - \\
\hline 9 & efGH & 11 & - & - & - & - & - & - & - & - \\
\hline 10 & efFH & 9.5 & - & - & & - & - & - & - & - \\
\hline 12 & $\mathrm{eb}^{\prime} \mathrm{EA}^{\prime}$ & 8.5 & 0.52 & 5.2 & $\mathrm{eb}^{\prime} \mathrm{EA}^{\prime} \mathrm{GH}$ & - & - & - & - & - \\
\hline 13 & $\mathrm{eb}^{\prime} \mathrm{FA}^{\prime}$ & 8.5 & 0.52 & 4.9 & $\mathrm{eb}^{\prime} \mathrm{FA}^{\prime} \mathrm{GH}$ & 0.64 & $>2.5$ & - & - & - \\
\hline
\end{tabular}

served alignment frequency favors the $[2,6]$ assignment [21]. The difference may also be due to remnants of pairing at high spins. If pairing is not negligible, $[(2) 2,6]$ is a six-quasiparticle configuration while $[2,6]$ is a four-quasiparticle configuration. The extra energy necessary to break a pair in order to form a six-quasiparticle excitation may lift the $[(2) 2,6]$ configuration in reality to higher energy than predicted by the CNS calculations which neglect pairing. Thus, Band 1 probably corresponds to the $[2,6]$ configuration in the spin region between 22 and 34 , in agreement with the previous assignments [12]. The calculated shape parameters for this configuration are $\varepsilon_{2} \approx 0.24$ and $\gamma \approx 0^{\circ}$.

Above the $I^{\pi}=34^{+}$state, Band 1 becomes irregular and forks into three branches, see Fig. 1. This is the typical behavior expected for band termination. Terminating bands have first been observed in Dy-Er region [39]. However, there exist analogies between the $A=160$ and 125 mass regions [20]. As illustrated in Fig. 11, four protons in $h_{11 / 2}$ orbitals outside the $Z=64$ semi-closed core in ${ }^{156} \mathrm{Er}$ may be compared to four neutrons in $h_{11 / 2}$ orbitals in ${ }^{124} \mathrm{Ba}$ which has $N=68$ neutrons. Similarly, six neutrons above the $N=82$ closed core in ${ }^{156} \mathrm{Er}$ is analogous to six protons outside the $Z=50$ closed core in ${ }^{124} \mathrm{Ba}$. Thus, the observed fully aligned configuration $\pi\left(h_{11 / 2}\right)_{16}^{4} \otimes \nu\left[\left(h_{9 / 2}\right)_{8}^{2}\left(f_{7 / 2}\right)_{6}^{2}\left(i_{13 / 2}\right)_{12}^{2}\right]$ of the $I^{\pi}=42^{+}$state in ${ }^{156} \operatorname{Er}[40]$ 
is analogous to the configuration $\pi\left[\left(h_{11 / 2}\right)_{10}^{2}\left(g_{7 / 2} d_{5 / 2}\right)_{10}^{4}\right] \otimes \nu\left(h_{11 / 2}\right)_{16}^{4}$ with $I^{\pi}=36^{+}$in ${ }^{124} \mathrm{Ba}$.

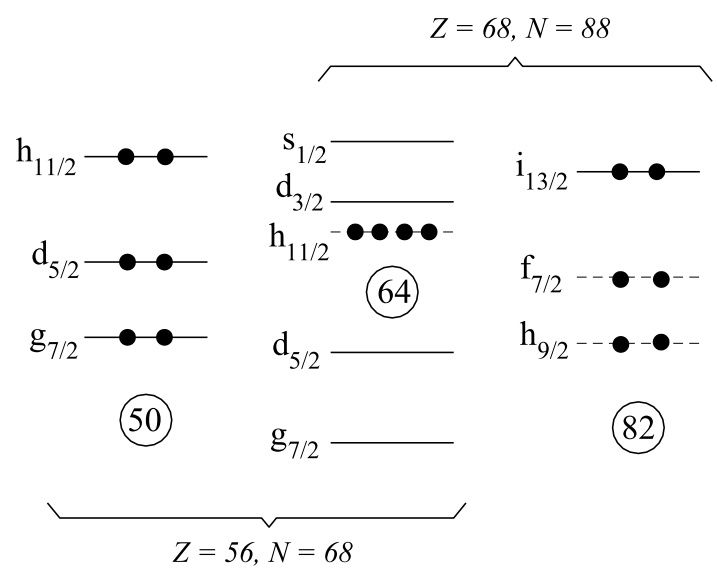

FIG. 11: Analogy between the $\mathrm{A}=125$ and 160 mass regions: the filling of subshells outside the ${ }^{114} \mathrm{Sn}$ and ${ }^{146} \mathrm{Gd}$ cores is illustrated schematically.

The present CNS calculations agree well with the expectations based on this analogy. In Fig. 6 we have differentiated between three types of states of the $[2,4]$ configuration defined above. The configuration $[2,4]$ a denotes the states with only four valence neutrons relative to the $N=64$ core and they all are located in the $h_{11 / 2}$ subshell, while $[2,4] \mathrm{b}$ and $[2,4] \mathrm{c}$ correspond to the states where one neutron and two neutrons, respectively, are excited from the $d_{5 / 2} g_{7 / 2}$ subshell to the $s_{1 / 2} d_{3 / 2}$ orbitals. The $[2,4]$ a configuration is predicted to become yrast around spin 34 and to terminate at $I^{\pi}=36^{+}$in a strongly favored state. Two favored $38^{+}$states and two favored $40^{+}$states are predicted corresponding to the $[2,4] \mathrm{b}$ and $[2,4]$ c configurations. Experimentally, we observe two $36^{+}$states and three $38^{+}$states. Comparing the relative positions of the calculated states with different configurations with the relative positions of the observed bands shown in Fig. 6, it is most likely that the $[2,4]$ a $\left(\pi\left[\left(h_{11 / 2}\right)_{10}^{2}\left(g_{7 / 2} d_{5 / 2}\right)_{10}^{4}\right] \otimes \nu\left(h_{11 / 2}\right)_{16}^{4}\right)$ terminating configuration has to be assigned to the yrast $36^{+}$state. This is a non-collective oblate state, characterized by the deformation parameters $\varepsilon_{2} \approx 0.2$ and $\gamma=60^{\circ}$. Its total angular momentum is built solely from the maximally aligned angular momenta of the valence particles corresponding to the $[2,4]$ a configuration. Comparing the relative positions of the higher-energy two nearly degenerate $36^{+}$states, they probably correspond to the $[2,6]$ and $[2,4] \mathrm{b}$ configurations, respectively. Similarly, the lowest two strongly favored $38^{+}$states probably correspond to the $[2,4] \mathrm{b}$ and $[2,4] \mathrm{c}$ configurations, respectively, while the highest-energy $38^{+}$state has the $[2,6]$ configuration. The $38^{+}$states belonging to $[2,4] \mathrm{b}$ and $[2,4] \mathrm{c}$ are partially aligned and these configurations are expected to terminate at spins 44 and 40, respectively. We note here that the predicted [2,4] structure 
in ${ }^{124} \mathrm{Ba}$ is very similar to the predicted [1,4] structure in ${ }^{123} \mathrm{Cs}[21]$, but with an additional $h_{11 / 2}$ proton. Therefore, the $36^{+}$state is analogous to the $63 / 2^{-}$terminating state in ${ }^{123} \mathrm{Cs}$. As shown in ref. [21], the states around this spin in ${ }^{123} \mathrm{Cs}$ are due to energy minima in the potential energy surface corresponding to zero, one and two particle-hole excitations from neutron $\left(d_{5 / 2} g_{7 / 2}\right)$ to $\left(s_{1 / 2} d_{3 / 2}\right)$ orbitals. Calculations for ${ }^{124} \mathrm{Ba}$ also show the existence of these minima around spin 36 .

Band 2 starts with an alignment of about $6.2 \hbar$, see Fig. 9, and shows a band crossing with an alignment gain of $4.4 \hbar$ at a frequency of $0.44 \mathrm{MeV} / \hbar$. Previously, the two-quasineutron configuration EF, of $\nu h_{11 / 2}$ origin, was assigned to this band below $0.44 \mathrm{MeV} / \hbar$. At this frequency the decoupling of an $h_{11 / 2}$ quasiproton pair takes place and the configuration changes to the two-quasineutron-two-quasiproton $\left(\nu h_{11 / 2}^{2} \otimes \pi h_{11 / 2}^{2}\right)$ structure, EFef [12]. In this frequency range, Band 1 has the same configuration, however in Band 2 the $h_{11 / 2}$ quasineutron pair is almost fully aligned $(\approx 6.2 \hbar)$ and the $h_{11 / 2}$ quasiproton pair is only partially aligned $(4.4 \hbar)$, whereas in Band 1 it is opposite: the $h_{11 / 2}$ quasiproton pair is nearly fully aligned $(8.2 \hbar)$ and the $h_{11 / 2}$ quasineutron pair shows a smaller alignment (3.3 $\hbar)$. The close similarity of the configurations of Bands 1 and 2 results in the strong decay from the higher-lying Band 2 into the yrast Band 1.

In the calculations using the CNS formalism the configurations above the first two band crossings in Bands 1 and 2 are the same, i.e. both are represented by a pair of aligned $h_{11 / 2}$ protons and $h_{11 / 2}$ neutrons, however with a different distribution of the valence protons and neutrons in low- $j$ orbitals. Indeed, a second minimum appears in the calculations with shape parameters $\varepsilon_{2} \approx 0.24$ and $\gamma \approx-30^{\circ}$. The corresponding configuration is labeled $[2,6]^{*}$ in Fig. 6. This minimum becomes more and more shallow with increasing spin and disappears above spin 34. However, the calculated relative energy difference between the first and second minimum does not agree well with that observed for Bands 1 and 2. At spin 22 , both the calculated and experimental energy differences are $\approx 250 \mathrm{keV}$. This difference is predicted to increase with increasing spin up to $\approx 700 \mathrm{keV}$, while experimentally it is constant up to $I=28$ and continuously decreases above this spin to about $30 \mathrm{keV}$. This behavior indicates a change in the configuration around spin 28. According to Fig. 6, a possible candidate for the configuration above this spin can be $[2,4] \mathrm{b}$ which is predicted to cross the $[2,6]$ configuration at spin 36 .

The alignment of Band 2, see Fig. 9, shows an irregularity at $\hbar \omega=0.63 \mathrm{MeV}$ with an alignment gain of $2.5 \hbar$, which is not seen in Band 1. In the yrast bands of the neighboring even-even nuclei ${ }^{126} \mathrm{Ba}$ [13] and ${ }^{128} \mathrm{Ba}$ [15] a similar behavior with alignment gains of $\approx 2$ and $\approx 4 \hbar$, respectively, has been observed. The alignment of a pair of protons of $g_{7 / 2}$ origin was suggested for the crossing around $\hbar \omega=0.6 \mathrm{MeV}$ in ${ }^{126} \mathrm{Ba}$ [13], whereas an alignment of 
a second pair of $h_{11 / 2}$ neutrons was proposed for the crossing at this frequency in ${ }^{128} \mathrm{Ba}[15]$. These may be alternative explanations for the behavior of Band 2 at high spins in ${ }^{124} \mathrm{Ba}$.

\section{B. Coupled Bands}

Information about the quasiparticle configurations of the coupled bands can be extracted from the ratios of reduced magnetic dipole and electric quadrupole transition probabilities, $B(M 1 ; I \rightarrow I-1) / B(E 2 ; I \rightarrow I-2)$. The experimental ratios have been obtained from the $\gamma$-ray-intensity ratios using

$$
\frac{B(M 1 ; I \rightarrow I-1)}{B(E 2 ; I \rightarrow I-2)}=0.697 \frac{I_{\gamma}(M 1 ; I \rightarrow I-1)}{I_{\gamma}(E 2 ; I \rightarrow I-2)} \frac{E_{\gamma}^{5}(E 2)}{E_{\gamma}^{3}(M 1)} \frac{1}{1+\delta^{2}}\left(\frac{\mu_{N}}{e b}\right)^{2}
$$

where $E_{\gamma}$ and $I_{\gamma}$ represent the energy in $\mathrm{MeV}$ and the intensity of the $\gamma$-ray transitions, respectively. The mixing ratio, $\delta$, for the $\Delta I=1$ transitions is small and $\delta^{2}$ has been set to zero. The experimentally deduced $B(M 1) / B(E 2)$ ratios are compared with the theoretical values obtained using the following generalized expression for multi-quasiparticle configurations, formulated in $[4,41]$ and based on the geometrical model of Dönau and Frauendorf $[42,43]$ :

$$
\begin{gathered}
\frac{B(M 1 ; I \rightarrow I-1)}{B(E 2 ; I \rightarrow I-2)}=\frac{12}{5 Q_{0}^{2} \cos ^{2}\left(\gamma+30^{\circ}\right)}\left[1-\frac{K_{\text {tot }}^{2}}{\left(I-\frac{1}{2}\right)^{2}}\right]^{-\mathbf{2}} \\
\times\left\{\left(I-\frac{K_{\text {tot }}^{2}}{I^{2}}\right)^{1 / 2}\left[K_{1}\left(g_{1}-g_{R}\right)\left(1 \pm \frac{\Delta e^{\prime}}{\hbar \omega}\right)+\sum_{\lambda} K_{\lambda}\left(g_{\lambda}-g_{R}\right)\right]\right. \\
\left.-\frac{K_{\text {tot }}}{I}\left[\left(g_{1}-g_{R}\right) i_{1}+\sum_{\lambda}\left(g_{\lambda}-g_{R}\right) i_{\lambda}\right]\right\}^{2} .
\end{gathered}
$$

$K_{\lambda}, g_{\lambda}$ and $i_{\lambda}$ stand for the $K$-value, gyromagnetic factor and aligned angular momentum, respectively, of the rotation- or Fermi-alinged quasiparticles involved in the configuration. $K_{1}, g_{1}$ and $i_{1}$ refer to the strongly coupled particle. The approximation $g_{R}=Z / A$ was used for the rotational $\mathrm{g}$ factor. $K_{\text {tot }}=K_{1}+\sum_{\lambda} K_{\lambda}$ denotes the total K-value of the configuration. The values for the intrinsic $\mathrm{g}$ factors for the different orbitals, $g_{\lambda}$, as well as the quadrupole moment, $Q_{\circ}=3.9 \mathrm{~b}$, have been taken from [13]. The shape parameter $\gamma$ was set to zero. The aligned angular momenta, $i_{m}$, were determined from the alignment plots, see Fig. 9. The signature splitting, $\Delta e^{\prime}$, was extracted from the routhian plots, see Fig. 10. The parameters used in the calculations are listed in Table IV. 
Bands 3 and 4 appear to be a pair of signature-partner bands with a large splitting. It was suggested [12] that Band 3 (the favored signature) corresponds to the quasiproton eb configuration and Band 4 (the unfavored signature) to ea or ec. The $\pi h_{11 / 2} g_{7 / 2}$ and $\pi\left(h_{11 / 2} d_{5 / 2}\right) \otimes \nu h_{11 / 2}^{4}$ configurations were also assigned to similar bands in the neighboring even-even nuclei ${ }^{126} \mathrm{Ba}[13]$ and ${ }^{128} \mathrm{Ba}[15]$, respectively. It was suggested [32, 33, 44] that those bands have a two-quasiproton structure mixed with the octupole vibrational band which accounts for the large signature splitting at low spins. Both bands are crossed by the configuration with an additional $h_{11 / 2}$ quasineutron pair, EF.

TABLE IV: Gyromagnetic factors and alignments used for the calculation of $B(M 1) / B(E 2)$ ratios.

\begin{tabular}{|c|c|c|c|c|c|}
\hline Bands & Subshell & Nilsson orbital & $g$-factor & $\mathrm{K}$ & $i[\hbar]$ \\
\hline \multirow[t]{3}{*}{3,4} & $\pi h_{11 / 2}$ & {$[550] \frac{1}{2}^{-}$} & 1.17 & 0.5 & 5.0 \\
\hline & $\pi g_{7 / 2}$ & {$[422] \frac{3}{2}^{+}$} & 0.72 & 1.5 & 1.0 \\
\hline & $\pi d_{5 / 2}$ & {$[420] \frac{1}{2}^{+}$} & 1.38 & 0.5 & 1.0 \\
\hline \multirow[t]{2}{*}{5,6} & $\pi h_{11 / 2}$ & {$[550] \frac{1}{2}^{-}$} & 1.17 & 0.5 & 5.0 \\
\hline & $\pi g_{9 / 2}$ & {$[404] \frac{9}{2}^{+}$} & 1.27 & 4.5 & 1.0 \\
\hline \multirow[t]{8}{*}{12,13} & $\pi h_{11 / 2}$ & {$[550] \frac{1}{2}^{-}$} & 1.17 & 0.5 & 5.0 \\
\hline & $\pi d_{5 / 2}$ & {$[420] \frac{1}{2}^{+}$} & 1.38 & 0.5 & 1.0 \\
\hline & $\pi g_{9 / 2}$ & {$[404] \frac{9}{2}^{+}$} & 1.27 & 4.5 & 0.0 \\
\hline & $\nu h_{11 / 2}$ & {$[532] \frac{5}{2}^{-}$} & -0.21 & 2.5 & 3.5 \\
\hline & & {$[541] \frac{3}{2}^{-}$} & -0.21 & 1.5 & 3.5 \\
\hline & $\nu g_{7 / 2}$ & {$[402] \frac{5}{2}^{+}$} & 0.21 & - & 0.5 \\
\hline & & {$[404] \frac{7}{2}^{+}$} & 0.21 & 3.5 & 0.5 \\
\hline & $\nu d_{5 / 2}$ & {$[413] \frac{5}{2}^{+}$} & -0.33 & 2.5 & 0.5 \\
\hline
\end{tabular}

The $B(M 1) / B(E 2)$ ratios determined from our data for Bands 3 and 4 are compared with the calculated values for the configurations $\pi h_{11 / 2} g_{7 / 2}$ and $\pi h_{11 / 2} d_{5 / 2}$ in Fig. 12. The calculated ratios for the configuration $\pi h_{11 / 2} d_{5 / 2}$ agree better with the experimental data than those 
for the $\pi h_{11 / 2} g_{7 / 2}$ configuration. Therefore, we have assigned the configuration $\pi h_{11 / 2} d_{5 / 2}$ to Bands 3 and 4 in the low-spin region. At higher spins these configurations are expected to become more and more mixed.

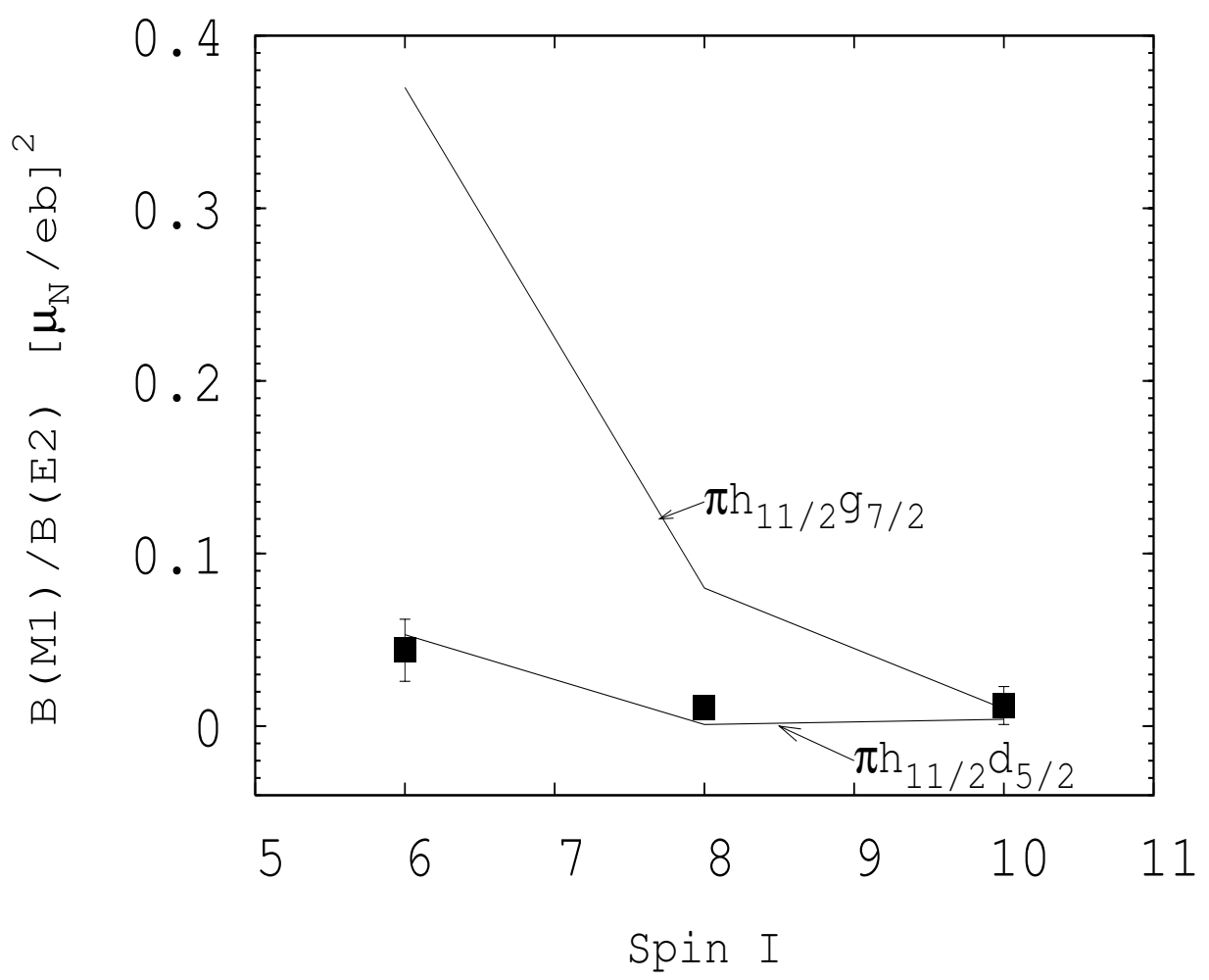

FIG. 12: $B(M 1) / B(E 2)$ ratios derived from measured $\gamma$-ray branching ratios for Bands 3 and 4 and calculated values for the $\pi h_{11 / 2} d_{5 / 2}$ and $\pi h_{11 / 2} g_{7 / 2}$ configurations.

Both bands show a band crossing at a rotational frequency of $\approx 0.45 \mathrm{MeV} / \hbar$ with a gain in alignment of 6 and $4 \hbar$, respectively. The rather large alignment suggests that aligned $h_{11 / 2}$ neutrons or protons are present in the configuration above that frequency. We suggest the configurations ebEF and eaGH for Bands 3 and 4, respectively, in the frequency range around $0.5 \mathrm{MeV} / \hbar$. At higher frequencies, Band 3 seems to loose alignment, see Fig. 9. However, this might be caused by a change to a reference core with a smaller deformation. A reduction of the Harris parameters to $\mathcal{J}_{\circ}=13 \hbar^{2} \mathrm{MeV}^{-1}$ and $\mathcal{J}_{1}=21 \hbar^{4} \mathrm{MeV}^{-3}$ makes the alignment of Band 3 constant.

Band 4 shows a gain in alignment of $\approx 6 \hbar$ at a frequency of $0.59 \mathrm{MeV} / \hbar$, see Fig. 9. Such a large gain can only be caused by $h_{11 / 2}$ neutrons or protons. As Band 3 does not show a similar increase at that frequency, the corresponding alignment has to be blocked. Thus, it is most likely that it is caused by a pair of $h_{11 / 2}$ neutrons, e.g. EF, which are already aligned in Band 3 at this frequency. At the highest observed frequencies, the configuration 
eaGHEF is therefore suggested for Band 4.

At high-spins the $[1,6]$ configuration is suggested by the CNS calculations. As seen in Fig. 7, there is good agreement for the relative positions and slopes between the calculated $[1,6]$ bands and the observed bands 3 and 4 up to spin 26 .

Band 14 decays into Band 3 around spin 30. Unfortunately, its spin assignment is not certain. If our tentative assignment is correct, Band 14 has an alignment which is $\approx 5 \hbar$ higher than that of Band 3, which could be explained by a six-quasiparticle configuration.

\section{Bands 5 and 6}

Bands 5 and 6 form a pair of signature partners with negligible signature splitting. The two-quasiproton configurations, $\mathrm{eb}^{\prime}$ and $\mathrm{ea}^{\prime}$, of $g_{9 / 2} h_{11 / 2}$ origin, seem to be a natural choice for these bands [12]. At a frequency of $0.44 \mathrm{MeV} / \hbar$ a band crossing with an alignment gain of $>6.1$ and $>5.8 \hbar$ is observed for Bands 5 and 6 , respectively. The high-spin part of the bands probably contains a pair of decoupled $h_{11 / 2}$ neutrons, resulting in the configurations $\mathrm{eb}^{\prime} \mathrm{EF}$ and ea'EF, respectively.

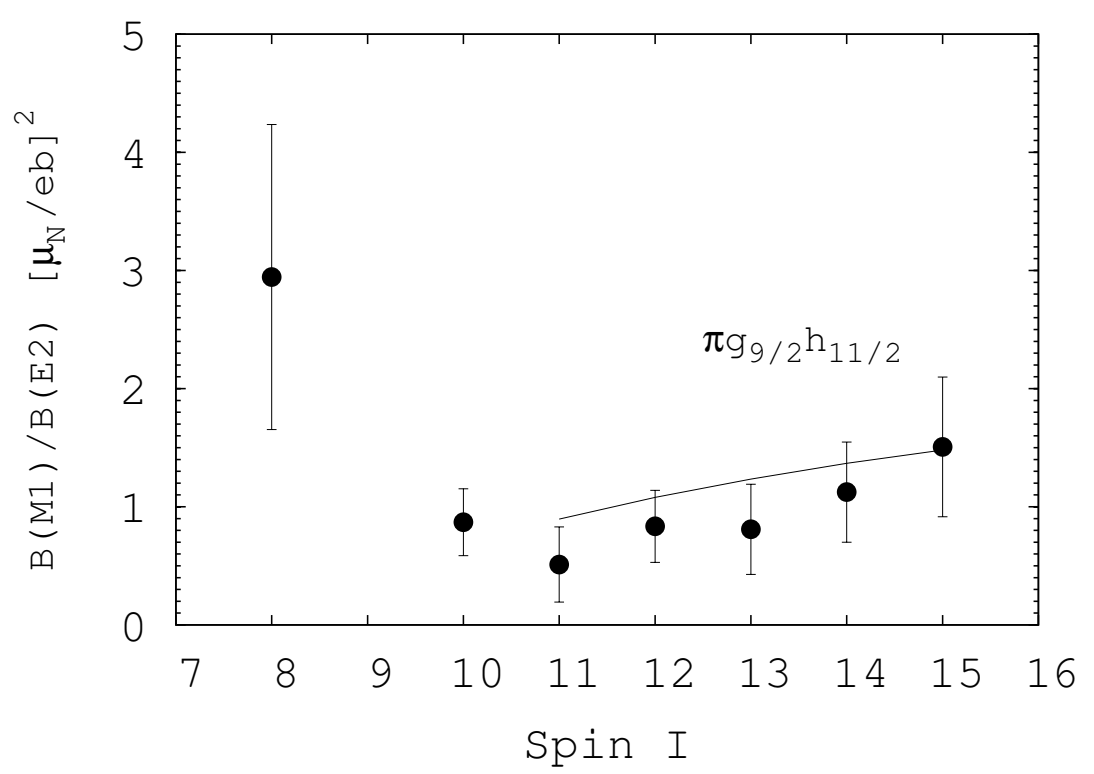

FIG. 13: $B(M 1) / B(E 2)$ ratios derived from measured $\gamma$-ray branching ratios for Bands 5 and 6 and calculation for the $\pi g_{9 / 2} h_{11 / 2}$ configuration.

$B(M 1) / B(E 2)$ ratios have been determined and are compared with calculated ratios for the $\pi g_{9 / 2} h_{11 / 2}$ configuration in Fig. 13. At lower spins a rather large $B(M 1) / B(E 2)$ ratio has 
been obtained. In this region mixing occurs with other states of the same spin and parity which also accounts for the observed decay-out transitions. Large $B(M 1) / B(E 2)$ ratios are calculated for configurations involving neutrons, e.g. for the $\nu h_{11 / 2} g_{7 / 2}$ or $\nu h_{11 / 2} s_{1 / 2}$ configurations. Mixing with such states may explain the observed increase of the $B(M 1) / B(E 2)$ ratios near the bottom of Bands 5 and 6 . The good agreement at medium spins supports the previous configuration assignment [12] which corresponds to the [(1)1,6] CNS configuration. The theoretically predicted relative positions and slopes of this configuration are in agreement with those of Bands 5 and 6, as seen in Fig. 7.

\section{Bands 7 and 8}

Bands 7 and 8 have been interpreted previously to be built on a $\gamma$ vibration $[18,45]$. The present results do not add significant information to these bands.

\section{Bands 12 and 13}

The new Bands 12 and 13 form a pair of coupled bands without signature splitting. Their alignment is rather large, $i_{x} \geq 8 \hbar$, already at low rotational frequencies, suggesting a multi-quasiparticle configuration. The experimental $B(M 1) / B(E 2)$ ratios determined from our data are compared with calculations for three different configurations, $\pi\left(h_{11 / 2} d_{5 / 2}\right) \otimes$ $\nu\left(h_{11 / 2} g_{7 / 2}\right), \pi\left(g_{9 / 2} h_{11 / 2}\right) \otimes \nu\left(h_{11 / 2} g_{7 / 2}\right)$ and $\pi\left(g_{9 / 2} h_{11 / 2}\right) \otimes \nu\left(h_{11 / 2} d_{5 / 2}\right)$, in Fig. 14. As can be seen, the calculated ratios for the configurations $\pi\left(g_{9 / 2} h_{11 / 2}\right) \otimes \nu\left(h_{11 / 2} g_{7 / 2}\right)$ and $\pi\left(g_{9 / 2} h_{11 / 2}\right) \otimes$ $\nu\left(h_{11 / 2} d_{5 / 2}\right)$ are close to the experimental data. These configurations are expected to be mixed and it is not possible distinguish between them. Thus, we assign the configurations eb'EA' and eb'FA' to Bands 12 and 13, respectively.

At a frequency of $\omega=0.5 \mathrm{MeV} / \hbar$ a further band crossing is observed. The alignment gain of about $5 \hbar$ may be due to a pair of $h_{11 / 2}$ neutrons. As orbitals $\mathrm{E}$ and $\mathrm{F}$ are already occupied, we suggest that the alignment is due to the GH quasineutron pair.

Two band structures with small signature splitting, the $[1,5]$ and $[(1) 2,6]$ configurations, are predicted by the present CNS calculations. They approach the yrast $[2,6]$ configuration in the spin 20 - 35 region, see Fig. 6. The experimental energy difference between the $[1,5]$ and $[2,6]$ configurations is predicted to decrease up to about spin 32 and then to increase at higher spins. For the [(1)2,6] configuration this energy difference is predicted to increase continuously in that spin region. The energy difference between Band 1 and Bands 12 and 13 decreases above spin 24 and becomes very small in the spin 30 - 35 region. This observation 


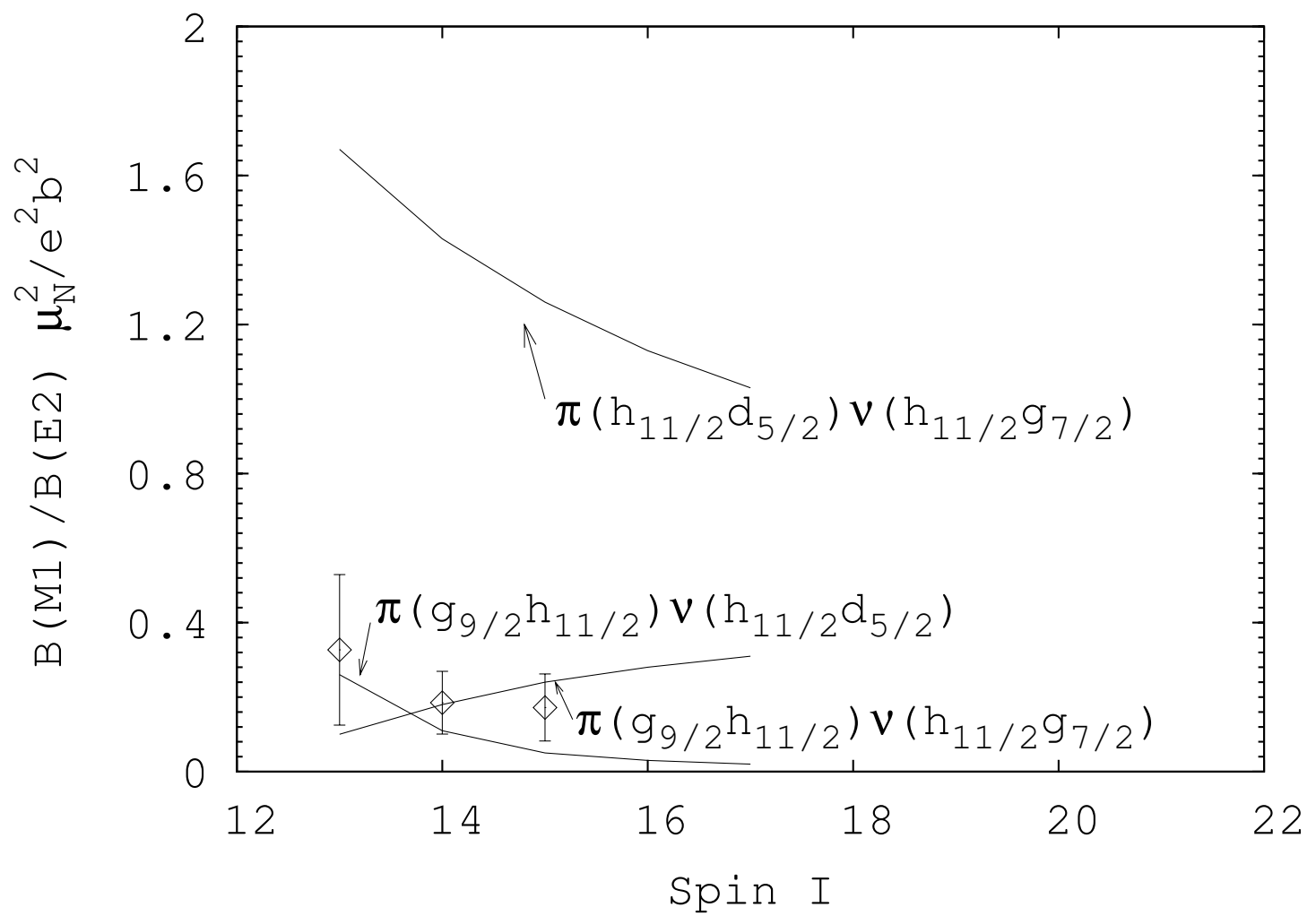

FIG. 14: $B(M 1) / B(E 2)$ ratios derived from measured $\gamma$-ray branching ratios for Bands 12 and 13 and calculated ratios for three different configurations.

favors the $[1,5]$ assignment over the $[(1) 2,6]$ configuration. Another argument against the $[(1) 2,6]$ configuration is that, similar to the case of the $[(2) 2,6]$ configuration discussed above, it may in reality lie at higher energy than predicted due to the pairing interaction which is not taken into account in the CNS calculations.

\section{Bands 9 and 10}

The new Bands 9 and 10 are observed above spins 17 and 18, respectively. They both decay into Band 1, suggesting a similarity in structure. They start with a high alignment which, in the lower part, increases by about $3 \hbar$ and then stays at 11 and $9.5 \hbar$, respectively, see Fig. 9. At higher spins their alignment pattern looks different. While the alignment of Band 9 decreases, Band 10 continuously gains alignment up to the highest-spin states observed. The rather large alignment suggests that both bands are four-quasiparticle configurations, probably containing a pair of aligned protons coupled to another aligned proton or a neutron pair. We tentatively assign the quasiparticle configurations efGH and efFH to Bands 9 and 
10, respectively. The difference in the appearance of the alignment might be caused by differences in the reference core. A reduction of the Harris parameters for Band 9 to $\mathcal{J}_{\circ}=13$ $\hbar^{2} \mathrm{MeV}^{-1}$ and $\mathcal{J}_{1}=21 \hbar^{4} \mathrm{MeV}^{-3}$ makes its alignment constant.

Inspection of the results of the CNS calculations presented in Fig. 6 shows that the configuration labeled $[1,5]$ could reproduce the observed properties reasonably well. However, it seems unlikely that Bands 9 and 10 are a pair of signature partners since no inter-band transitions are observed. Furthermore, we have already tentatively assigned this configuration to Bands 12 and 13, as discussed above. Thus, there are no good candidates predicted by the present CNS calculations for the configurations of these bands.

\section{Band 11}

Band 11 is not firmly connected to known levels in ${ }^{124} \mathrm{Ba}$. However, the systematics of excitation energies and spins of the bands observed in this nucleus allow an approximate placement in the level scheme and limit the spin range. As the band decays to high-spin states of Band 1, their structures should have some similarity.

Band 11 is observed only above spin 25 or 26 and continues without band crossing up to spin 45 or 46 . The most likely candidate from the CNS calculations is the [2,5(1)] configuration, i.e. $\pi h_{11 / 2}^{2} \otimes \nu h_{11 / 2}^{5} i_{13 / 2}$. In its decay to Band 1 it goes over to the $[2,6]$ configuration with two $h_{11 / 2}$ protons and six $h_{11 / 2}$ neutrons.

Band 11 shows a gradual decrease in the dynamic moment of inertia in a manner similar to that of several high-spin bands in this mass region. In Fig. 15 the dynamic moments of inertia of Band 11 and similar bands observed in ${ }^{132} \mathrm{Ce}$ [46-48] and ${ }^{126} \mathrm{Xe}$ [49] are compared. The ${ }^{132} \mathrm{Ce}$ band is highly deformed; a transition quadrupole moment of $Q=8.8$ b has been measured [46]. In ${ }^{125,126}$ Xe several bands, which extend up to very high spins, have been observed recently [49]. However, only for the ${ }^{126}$ Xe band shown in Fig. 15 was the quadrupole moment estimated, $Q \sim 5.2 \mathrm{~b}$ [49]. The dynamic moment of inertia of Band 11 lies between those of ${ }^{126} \mathrm{Xe}$ and ${ }^{132} \mathrm{Ce}$ shown in Fig. 15. This systematics suggests that it probably has a larger deformation than the other bands in ${ }^{124} \mathrm{Ba}$. The excitation of an $i_{13 / 2}$ neutron may cause the larger deformation, however lifetime measurements are needed for a final proof. CSM calculations for ${ }^{132}$ Ce show that the frequency dependence of the dynamic moment of inertia is a combined effect of gradual alignment of protons in $h_{11 / 2}$ and neutrons in $h_{9 / 2}$ and $h_{11 / 2}$ orbitals [50], and a similar explanation may apply also to Band 11 in ${ }^{124} \mathrm{Ba}$. 


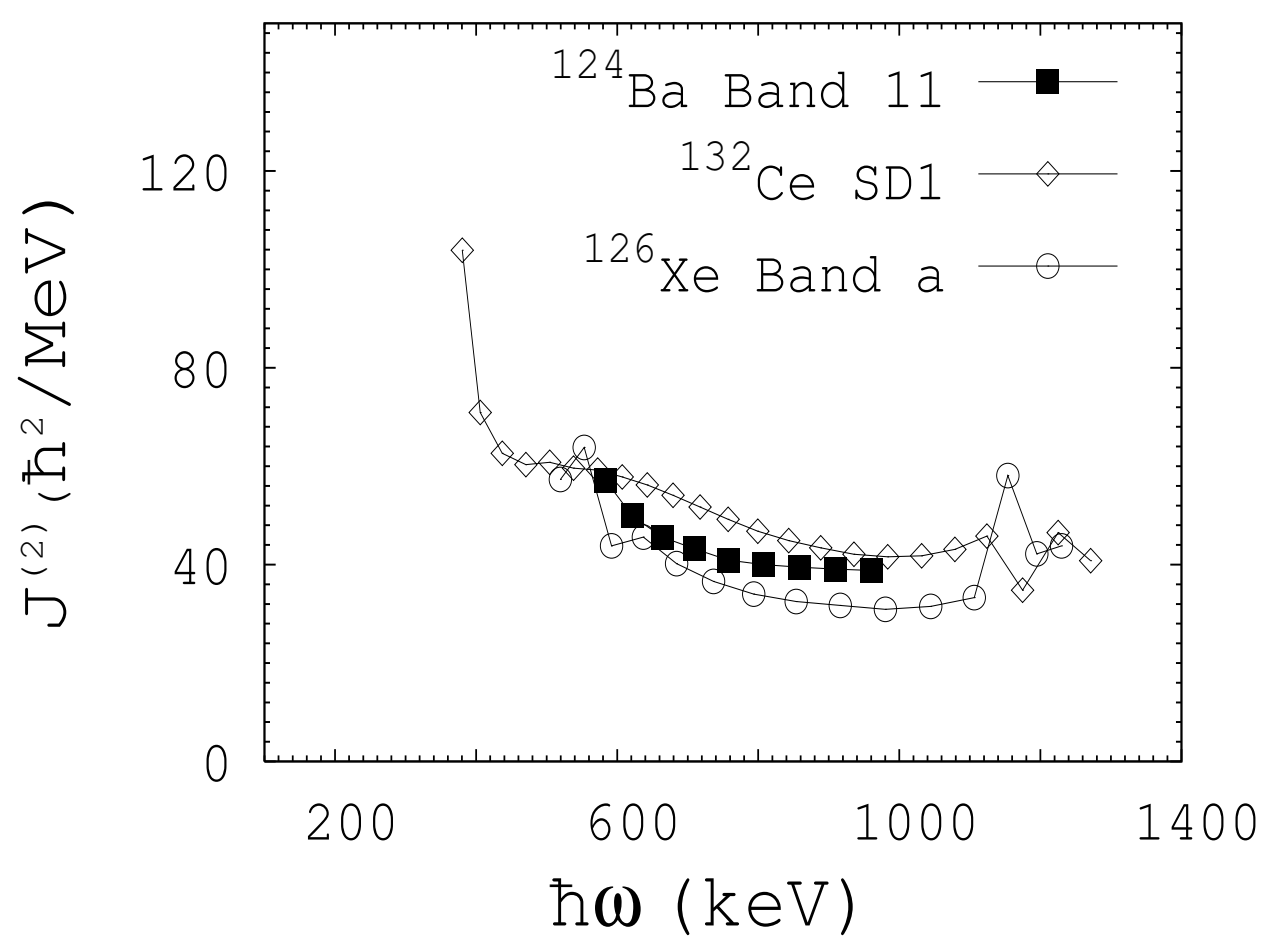

FIG. 15: Dynamic moment of inertia, $\mathcal{J}^{(2)}$, for Band 11 in ${ }^{124} \mathrm{Ba}$ as well as for similar bnds in ${ }^{132} \mathrm{Ce}[48]$ and ${ }^{126} \mathrm{Xe}[49]$, as a function of rotational frequency.

\section{SUMMARY}

In summary, high-spin states in ${ }^{124} \mathrm{Ba}$ have been investigated in two experiments using the Euroball and Gammasphere spectrometers, respectively. Previously known bands have been extended and six new bands have been found. Configurations have been assigned to the band structures, based on comparisons with predictions of the CSM in the lower-spin region and with the CNS model at high spins. The positive-parity yrast band, Band 1, shows the typical behavior of band termination at the highest spins. Configurations for the terminating states which compete with the collective excitations are suggested on tha basis of CNS calculations. One of the new bands, Band 11, extends to spins around 46 without band crossings. Its dynamic moment of inertia decreases steadily, similar to those of large-deformed bands in this mass region. 


\section{ACKNOWLEDGMENTS}

This work was supported in part by the EU (contract EUROVIV: HPRI-CT-1999-00078), the German BMBF (contract 06 BN 907), the Hungarian Scientific Research Fund OTKA (contract numbers T046901 and T038404), the Bolyai János Foundation of HAS, and by INFN, Italy. The authors are grateful to I. Ragnarsson for helpful discussions of the results of the CSN calculations and the configuration assignments to the band structures in ${ }^{124} \mathrm{Ba}$.

[1] C. M. Petrache, G. LoBianco, P. G. Bizzeti, A. M. Bizzeti-Sona, D. Bazzacco, S. Lunardi, M. Nespolo, G. de Angelis, D. R. Napoli, N. Blasi, et al., Eur. Phys. J. A 16, 337 (2003).

[2] J. F. Smith, V. Medina-Chico, C. J. Chiara, M. P. Carpenter, C. N. Davids, M. Devlin, J. L. Durell, D. B. Fossan, S. J. Freeman, R. V. F. Janssens, et al., Phys. Rev. C 69, 034339 (2004).

[3] F. Seiffert, W. Lieberz, A. Dewald, S. Freund, A. Gelberg, A. Granderath, D. Lieberz, R. Wirowski, and P. von Brentano, Nucl. Phys. A 554, 287 (1993).

[4] S. Törmänen, S. Juutinen, R. Julin, B. Cederwall, A. Johnson, R. Wyss, P. Ahonen, B. Fant, M. Matsuzaki, J. Nyberg, et al., Nucl. Phys. A 572, 417 (1994).

[5] H. Timmers, J. Simpson, M. A. Riley, T. Bengtsson, M. A. Bentley, F. Hanna, S. M. Mullins, J. F. Sharpey-Schafer, and R. Wyss, J. Phys. G: Nucl. Part. Phys. A 20, 287 (1994).

[6] J. Timár, J. Simpson, E. S. Paul, S. Araddad, C. W. Beausang, M. A. Bentley, M. J. Joyce, and J. F. Sharpey-Schafer, J. Phys. G: Nucl. Part. Phys. 21, 783 (1995).

[7] I. Wiedenhöver, J. Yan, U. Neuneyer, R. Wirowski, P. von Brentano, A. Gelberg, N. Yoshida, and T. Otsuka, Nucl. Phys. A 582, 77 (1995).

[8] M. Serris, C. T. Papadopoulos, R. Vlastou, C. A. Kalfas, S. Kossionides, N. Fotiades, S. Harissopulos, C. W. Beausang, M. J. Joyce, E. S. Paul, et al., Z. Phys. A 358, 37 (1997).

[9] I. Schneider, R. S. Chakrawarthy, I. Wiedenhöver, A. Schmidt, H. Meise, P. Petkov, A. Dewald, P. von Brentano, O. Stuch, K. Jessen, et al., Phys. Rev. C 60, 014312 (1999).

[10] V. Werner, H. Meise, I. Wiedenhöver, A. Gade, and P. von Brentano, Nucl. Phys. A 692, 451 (2001).

[11] R. Wyss, A. Granderath, R. Bengtsson, P. von Brentano, A. Dewald, A. Gelberg, A. Gizon, J. Gizon, S. Harissopulos, A. Johnson, et al., Nucl. Phys. A 505, 337 (1989). 
[12] S. Pilotte, S. Flibotte, S. Monaro, N. Nadon, N. Prevost, P. Taras, H. R. Andrews, D. Horn, V. P. Janzen, D. C. Radford, et al., Nucl. Phys. A 514, 545 (1990).

[13] D. Ward, V. P. Janzen, H. R. Andrews, D. C. Radford, G. C. Ball, D. Horn, J. C. Waddington, J. K. Johansson, F. Banville, J. Gascon, et al., Nucl. Phys. A 529, 315 (1991).

[14] A. Granderath, P. F. Mantica, R. Bengtsson, R. Wyss, P. von Brentano, A. Gelberg, and F. Seiffert, Nucl. Phys. A 597, 427 (1996).

[15] O. Vogel, R. S. Chakrawarthy, A. Dewald, P. Petkov, K. Jessen, J. Gableske, P. von Brentano, D. Bazzacco, A. Gizon, J. Gizon, et al., Eur. Phys. J. A 4, 323 (1999).

[16] J. F. Smith, C. J. Chiara, D. B. Fossan, G. J. Lane, J. M. Sears, I. Thorslund, I. M. Hibbert, R. Wadsworth, I. Y. Lee, and A. O. Macchiavelli, Phys. Lett. B 483, 7 (2000).

[17] C. M. Petrache, G. LoBianco, D. Bazzacco, T. Kröll, S. Lunardi, R. Menegazzo, M. Nespolo, P. Pavan, C. R. Alvarez, G. de Angelis, et al., Eur. Phys. J. A 12, 135 (2001).

[18] J. P. Martin, V. Barci, H. El-Samman, A. Gizon, J. Gizon, W. Klamra, B. M. Nyakó, F. A. Beck, T. Byrski, and J. C. Merdinger, Nucl. Phys. A 489, 169 (1988).

[19] A. V. Afanasjev, D. B. Fossan, G. J. Lane, and I. Ragnarsson, Phys. Rep. 322, 1 (1999).

[20] I. Ragnarsson, Z. Xing, T. Bengtsson, and M. A. Riley, Phys. Scripta Vol. 34, 651 (1986).

[21] A. K. Singh, H. Hübel, J. Domscheit, G. B. Hagemann, B. Herskind, D. R. Jensen, J. N. Wilson, R. Clark, M. Cromaz, P. Fallon, et al., Phys. Rev. C 70, 034315 (2004).

[22] A. Al-Khatib, A. K. Singh, H. Hübel, P. Bringel, A. Bürger, A. Neußer-Neffgen, G. Schönwaßer, G. Hagemann, C. R. Hansen, B. Herskind, et al., Acta Phys. Pol. B 36, 1029 (2005).

[23] H. Hübel, Acta Phys. Pol. B 36, 1015 (2005).

[24] I. Y. Lee, Nucl. Phys. A 520, 641c (1990).

[25] F. A. Beck, Prog.Part.Nucl.Phys. 28, 443 (1992).

[26] J. Simpson, Z. Phys. A 358, 139 (1997).

[27] J. N. Scheurer, M. Aiche, M. M. Aleonard, G. Barreau, F. Bourgine, D. Boivin, D. Cabaussel, J. F. Chemin, T. P. Doan, J. P. Goudour, et al., Nucl. Instr. Meth. A 385, 501 (1997).

[28] J. Gál, G. Hegyesi, J. Molnár, B. M. Nyakó, G. Kalinka, J. N. Scheurer, M. M. Aléonard, J. F. Chemin, J. L. Pedroza, K. Juhász, et al., Nucl. Instr. Meth. A 516, 502 (2004).

[29] D. C. Radford, Nucl. Instr. Meth. A 361, 297 (1995).

[30] O. Klein and Y. Nishina, Z. Phys. 52, 853 (1929). 
[31] D. Roßbach, A. Görgen, H. Hübel, E. Mergel, G. Schönwaßer, A. N. Wilson, F. Azaiez, A. Astier, D. Bazzacco, M. Bergström, et al., Phys. Lett. B 513, 9 (2001).

[32] P. Mason, G. Benzoni, A. Bracco, F. Camera, B. Million, O. Wieland, S. Leoni, A. K. Singh, A. Al-Khatib, H. Hübel, et al., Phys. Rev. C 72, 064315 (2005).

[33] R. Piepenbring and J. Leandri, Phys. Lett. B 267, 17 (1991).

[34] R. Bengtsson and S. Frauendorf, Nucl. Phys. A 314, 27 (1979).

[35] R. Bengtsson, S. Frauendor, and F. R. May, At. Nucl. Data Tables 35, 15 (1986).

[36] T. Bengtsson and I. Ragnarsson, Nucl. Phys. A 436, 14 (1985).

[37] A. V. Afanasjev and I. Ragnarsson, Nucl. Phys. A 608, 176 (1996).

[38] D. M. Todd, R. Aryaeinejad, D. J. G. Love, A. H. Nelson, P. J. Nolan, P. J. Smith, , and P. J. Twin, J. Phys. G: Nucl. Part. Phys. 10, 1407 (1984).

[39] A. V. Afanasjev and I. Ragnarsson, Nucl. Phys. A 591, 387 (1995).

[40] F. S. Stephens, M. A. Delplanque, R. M. Diamond, A. O. Macchiavelli, and J. E. Draper, Phys. Rev. Lett. 54, 2584 (1985).

[41] D. C. Radford, H. R. Andrews, G. C. Ball, D. Horn, D. Ward, F. Banville, S. Flibotte, S. Monaro, S. Pilotte, P. Taras, et al., Nucl. Phys. A 545, 665 (1992).

[42] F. Dönau and S. Frauendorf, Proc. Conf. on High Angular Momentum Propereties of Nuclei (Oak Ridge, 1982) ed. N.R. Johnson (New York: Harwood Academic) p. 143 (1983).

[43] F. Dönau, Nucl. Phys. A 471, 469 (1987).

[44] R. Wyss, A. Johnson, D. J. G. Love, M. J. Godfrey, and S. M. Mullins, Z. Phys. A 332, 241 (1989).

[45] T. Komatsubara, T. Hosoda, H. Sakamoto, T. Aoki, and K. Furuno, Nucl. Phys. A 496, 605 (1989)

[46] A. J. Kirwan, G. C. Ball, P. J. Bishop, M. J. Godfrey, P. J. Nolan, D. J. Thornley, D. J. G. Love, and A. H. Nelson, Phys. Rev. Lett. 58, 467 (1987).

[47] P. J. Nolan, A. Kirwan, D. J. G. Love, A. H. Nelson, D. J. Unwin, and P. J. Twin., J. Phys. G: Nucl. Part. Phys. 11, L17 (1985).

[48] E. S. Paul, P. T. W. Choy, C. Andreoiu, A. J. Boston, A. O. Evans, C. Fox, S. Gros, P. J. Nolan, G. Rainovski, J. A. Sampson, et al., Phys. Rev. C 71, 054309 (2005).

[49] C. R. Hansen, G. B. Hagemann, B. Herskind, D. R. Jensen, G. Sletten, J. N. Wilson, S. Ødegård, P. Bringel, C. Engelhardt, H. Hübel, et al., AIP Conf. Proc. 764, 46 (2005). 
[50] R. Wyss, J. Nyberg, A. Johnson, R. Bengtsson, and W. Nazarewicz, Phys. Lett. B 215, 211 (1988). 